\title{
Identification of Unknown Parameters and Orders via Cuckoo Search Oriented Statistically by Differential Evolution for Noncommensurate Fractional-Order Chaotic Systems
}

\author{
Fei Gao, ${ }^{1}$ Xue-Jing Lee, ${ }^{1}$ Heng-qing Tong, ${ }^{1}$ Feng-xia Fei, ${ }^{1}$ and Hua-ling Zhao ${ }^{2}$ \\ ${ }^{1}$ Department of Mathematics, School of Science, Wuhan University of Technology, Luoshi Road 122, Wuhan, Hubei 430070, China \\ ${ }^{2}$ Department of Statistics, School of Science, Wuhan University of Technology, Luoshi Road 122, Wuhan, Hubei 430070, China
}

Correspondence should be addressed to Fei Gao; hgaofei@gmail.com

Received 1 May 2013; Accepted 8 October 2013

Academic Editor: Abdelghani Bellouquid

Copyright (C) 2013 Fei Gao et al. This is an open access article distributed under the Creative Commons Attribution License, which permits unrestricted use, distribution, and reproduction in any medium, provided the original work is properly cited.

In this paper, a non-Lyapunov novel approach is proposed to estimate the unknown parameters and orders together for noncommensurate and hyper fractional chaotic systems based on cuckoo search oriented statistically by the differential evolution (CSODE). Firstly, a novel Gaos' mathematical model is proposed and analyzed in three submodels, not only for the unknown orders and parameters' identification but also for systems' reconstruction of fractional chaos systems with time delays or not. Then the problems of fractional-order chaos' identification are converted into a multiple modal nonnegative functions' minimization through a proper translation, which takes fractional-orders and parameters as its particular independent variables. And the objective is to find the best combinations of fractional-orders and systematic parameters of fractional order chaotic systems as special independent variables such that the objective function is minimized. Simulations are done to estimate a series of noncommensurate and hyper fractional chaotic systems with the new approaches based on CSODE, the cuckoo search, and Genetic Algorithm, respectively. The experiments' results show that the proposed identification mechanism based on CSODE for fractional orders and parameters is a successful method for fractional-order chaotic systems, with the advantages of high precision and robustness.

\section{Introduction}

The applications of fractional differential equations began to appeal to related scientists recently [1-27] in the following areas: bifurcation, hyperchaos, proper and improper fractional-order chaos systems, and chaos synchronization [1-33].

However, there are some systematic parameters and orders that are unknown for the fractional-order chaos systems in controlling and synchronization. It is difficult to identify the parameters in the fractional-order chaotic systems with unknown parameters. Hitherto, there have been two main approaches in parameters' identification for fractional-order chaos systems.

(i) Lyapunov way: there have been few results on parameter estimation method of fractional-order chaotic systems based on chaos synchronization [34] and methods for parameter estimation of uncertain fractional order complex networks [35]. However, the design of controller and the updating law of parameter identification are still a tough task with technique and sensitively depend on the considered systems.

(ii) Non-Lyapunov way via artificial intelligence methods, for examples, differential evolution [7] and particle swarm optimization [9], in which the commensurate fractional-order chaos systems and simplest case with one unknown order for normal fractional-order chaos systems are discussed: however, to the best of our knowledge, little work in non-Lyapunov way has been done to the parameters and orders estimation of noncommensurate and hyper fractional-order chaos systems. And there is no general mathematical model that has been purposed for all these kinds of identification.

We consider the following fractional-order chaos system with time delays

$$
{ }_{\alpha} \mathscr{D}_{t}^{q} Y(t)=f\left(Y(t), Y_{0}(t), \theta, \tau\right),
$$


where $Y(t)=\left(y_{1}(t), y_{2}(t), \ldots, y_{n}(t)\right)^{T} \in \mathfrak{R}^{n}$ denotes the state vector. $\theta=\left(\theta_{1}, \theta_{2}, \ldots, \theta_{n}\right)^{T}$ denotes the original parameters, $\tau=\left(\tau_{1}, \tau_{2}, \ldots, \tau_{n}\right)$ is the time delay, and $q=\left(q_{1}, q_{2}, \ldots, q_{n}\right)$ $\left(0<q_{i}<1, i=1,2, \ldots, n\right)$ is the fractional derivative orders. Consider

$$
\left.f\right|_{\left(Y(t), Y_{0}(t), \theta, \tau\right)}=\left.\left(f_{1}, f_{2}, \ldots, f_{n}\right)\right|_{\left(Y(t), Y_{0}(t), \theta, \tau\right)} .
$$

Normally the function $f$ is known. And the $\theta, q$, and $\tau$ are unknown; then $\Theta=\left(\theta_{1}, \theta_{2}, \ldots, \theta_{n}, q_{1}, q_{2}, \ldots, q_{n}, \tau_{1}\right.$, $\left.\tau_{2}, \ldots, \tau_{n}\right)$ will be the parameters to be estimated.

Then a correspondent system is constructed as follows

$$
{ }_{\alpha} \mathscr{D}_{t}^{q} \tilde{Y}(t)=f\left(\tilde{Y}(t), Y_{0}(t), \tilde{\theta}, \tilde{\tau}\right),
$$

where $\tilde{Y}(t), \tilde{\theta}, \tilde{q}$, and $\tilde{\tau}$ are the correspondent variables to those in (1), and function $f$ is the same. The two systems (1) and (3) have the same initial condition $Y_{0}(t)$.

Then the objective is obtained as follows:

$$
\Theta^{*}=\arg \min _{\Theta} F=\arg \min _{\Theta} \sum_{t}\|Y(t)-\tilde{Y}(t)\|_{2} .
$$

How could we identify the fractional system, when some fractional chaotic differential equations $f=\left(f_{1}, f_{2}, \ldots, f_{n}\right)$ are unknown? That is,

$$
\left(f_{1}, f_{2}, \ldots, f_{n}\right)^{*}=\arg \min _{\left(f_{1}, f_{2}, \ldots, f_{n}\right)} F .
$$

Now the problem of parameters estimation (4) becomes another much more complicated question, fractional-order chaos reconstruction problem [36], to find the forms of fractional-order equations as in (5). In [36] a novel non-Lyapunov reconstruction method based on a novel united mathematical model was proposed to reconstruct the unknown equations $\left(f_{1}, f_{2}, \ldots, f_{n}\right)$.

When it comes to the system (1) ${ }_{\alpha} \mathscr{D}_{t}^{q} Y(t)=$ $f\left(Y(t), Y_{0}(t), \theta\right)$ with neither $q$ nor $\theta$ are known, the united model is not effective. For the united mathematical model [36], to be identified is only $\left(f_{1}, f_{2}, \ldots, f_{n}\right)$ instead of $q$. That is, ${ }_{\alpha} \mathscr{D}_{t}^{q} \tilde{Y}(t)$ of (3) are not included. Actually, if the $q$ are taken into consideration in united model (5), then the basic parameters' setting to be reconstructed in [36] will be basic set $\{\times, \div,+,-\}$ with extra $\left\{=, \mathscr{D}_{t}^{q}\right\}$ and so forth and the input variables $\left\{x_{1}, x_{2}, \ldots, x_{n}\right\}$ with $\{Y\}$ extra and, and so forth. Although for the candidates "programs" in [36] the maximum depth of tree is 6 , considering that the maximum number of nodes per tree is infinite, there will be infinite illegal candidates on will be generated. Then, the one hand, the most time-consuming thing for novel united model (5) is to kill these illegal individuals from the legal individuals. However, these defaults are not solved in [36]. On the other hand, as $q \in \mathscr{D}_{t}^{q}$ in unknown, it is really difficult to generate an individual with $\left\{x, \div,+,-,=, \mathscr{D}_{t}^{q}\right\}$, in neither illegal nor legal cases. And up till now, there is no existing way to resolve these defaults. And we can conclude from simulations [36] that the proposed method is much more efficient for the systems when coefficients $q$ in $\mathscr{D}_{t}^{q}$ are integer orders than improper fractional orders.
Therefore, to estimate the $q$ of (3) with unknown systematic parameters $\theta$ is still a question to be solved for parameters and orders estimation of noncommensurate and hyperfractional-order chaos systems.

And cuckoo search (CS) is a relatively new and robust optimization algorithm [37, 38], inspired by the obligate brood parasitism of some cuckoo species by laying their eggs in the nests of other host birds (of other species). The searching performance is mainly based on the Lévy flights mathematically [37-39], which essentially provide a random walk while their random steps are drawn from a Lévy distribution for large steps [37-39]. However, in CS evolution, the Lévy flights in each main iteration are used twice. It has two results: the CS searching performance becomes a little strong, but the redundant evaluations for the objective function are generated too. Therefore, some more efforts are needed to improve the performance of CS.

To the best of authors' knowledge, there is no method in non-Lyapunov way for noncommensurate and hyper fractional-order chaotic systems' parameters and orders estimation so far. The objective of this work is to present a novel simple but effective approach to estimate the noncommensurate and hyper fractional-order chaotic systems in a nonLyapunov way. And the illustrative reconstruction simulations in different chaos systems are discussed, respectively.

The rest is organized as follows. In Section 2, a general mathematical model not only for fractional chaos parameters identification but also for reconstruction in non-Lyapunov way is newly proposed and analyzed in three submodels $\mathrm{A}, \mathrm{B}$, and $\mathrm{C}$. And a simple review was given on nonLyapunov parameters estimation methods for fractionalorder and normal chaos systems. In Section 3, a novel method with proposed united model based on cuckoo search oriented statistically by differential evolution (CSODE) is proposed. And simulations are done to a series of different noncommensurate and hyper fractional-order chaotic systems by a novel method based on CSODE, single cuckoo search, and Genetic Algorithm, respectively, in Section 4. Conclusions are summarized briefly in Section 5 .

\section{Gao's Mathematical Model for Fractional Chaos Reconstruction and Orders Estimation in Non-Lyapunov Way}

In this section, a general mathematical model for fractional chaos parameters identification in non-Lyapunov way is proposed. A detail explanation for the general mathematical model will be given in the following subsections in three aspects, submodel A, B, and C.

2.1. Gao's Mathematical Model. Now we consider the general forms of fractional-order chaos system (1). To make the system (1) more clear, we take its equivalent form as the following system:

$$
\begin{array}{r}
{ }_{\alpha} \mathscr{D}_{t}^{q_{1}} y_{1}(t)=f_{1}\left(t, y_{1}(t), y_{1}(t-\tau), y_{2}(t),\right. \\
\left.y_{2}(t-\tau), \ldots, y_{n}(t), y_{n}(t-\tau)\right),
\end{array}
$$




$$
\begin{gathered}
\alpha_{t}^{\mathscr{D}_{2}} y_{2}(t)=f_{2}\left(t, y_{1}(t), y_{1}(t-\tau), y_{2}(t),\right. \\
\left.y_{2}(t-\tau), \ldots, y_{n}(t), y_{n}(t-\tau)\right) \\
\vdots \\
\alpha_{t} \mathscr{D}_{t}^{q_{n}} y_{n}(t)=f_{n}\left(t, y_{1}(t), y_{1}(t-\tau), y_{2}(t),\right. \\
\left.y_{2}(t-\tau), \ldots, y_{n}(t), y_{n}(t-\tau)\right), \\
L=\left(y_{1}, y_{2}, \ldots, y_{n}\right) .
\end{gathered}
$$

And a correspondent system (7) is constructed as follow:

$$
\begin{aligned}
& { }_{\alpha} \mathscr{D}_{t}^{\widetilde{q}_{1}} \tilde{y}_{1}(t)=\tilde{f}_{1}\left(t, \tilde{y}_{1}(t), \tilde{y}_{1}(t-\widetilde{\tau}), \tilde{y}_{2}(t),\right. \\
& \left.\tilde{y}_{2}(t-\tilde{\tau}), \ldots, \tilde{y}_{n}(t), \tilde{y}_{n}(t-\tilde{\tau})\right), \\
& { }_{\alpha} \mathscr{D}_{t}^{\widetilde{q}_{2}} \widetilde{y}_{2}(t)=\tilde{f}_{2}\left(t, \tilde{y}_{1}(t), \tilde{y}_{1}(t-\tilde{\tau}), \widetilde{y}_{2}(t),\right. \\
& \left.\tilde{y}_{2}(t-\tilde{\tau}), \ldots, \tilde{y}_{n}(t), \tilde{y}_{n}(t-\tilde{\tau})\right) \\
& \alpha^{\tilde{D}_{t}} \tilde{y}_{n}(t)=\widetilde{f}_{n}\left(t, \tilde{y}_{1}(t), \tilde{y}_{1}(t-\tilde{\tau}), \tilde{y}_{2}(t),\right. \\
& \left.\tilde{y}_{2}(t-\tilde{\tau}), \ldots, \tilde{y}_{n}(t), \tilde{y}_{n}(t-\tilde{\tau})\right), \\
& \widetilde{L}=\left(\tilde{y}_{1}, \tilde{y}_{2}, \ldots, \tilde{y}_{n}\right) \text {. }
\end{aligned}
$$

To have simple forms, we take $\alpha=0$.

Then novel objective function (fitness) (8) in this paper comes into being from (20) and (21) as follows:

$$
F=\sum_{t=0 \cdot h}^{T \cdot h}\|\widetilde{L}-L\|_{2}
$$

where $T$ is the number of sample points for methods resolving the fractional systems and $h$ is the time step.

Now a novel Gao mathematical model for fractional chaos reconstruction comes into being as Figure 1 shows, where functions $f=\left(f_{1}, f_{2}, \ldots, f_{n}\right), \widetilde{f}=\left(\widetilde{f}_{1}, \widetilde{f}_{2}, \ldots, \widetilde{f}_{n}\right)$, fractional orders $q=\left(q_{1}, q_{2}, \ldots, q_{n}\right), \widetilde{q}=\left(\widetilde{q}_{1}, \widetilde{q}_{2}, \ldots, \widetilde{q}_{n}\right)$, time delays $\tau=\left(\tau_{1}, \tau_{2}, \ldots, \tau_{n}\right), \widetilde{\tau}=\left(\widetilde{\tau}_{1}, \widetilde{\tau}_{2}, \ldots, \widetilde{\tau}_{n}\right)$, and systematic parameters $\theta=\left(\theta_{1}, \theta_{2}, \ldots, \theta_{n}\right), \widetilde{\theta}=\left(\widetilde{\theta}_{1}, \widetilde{\theta}_{2}, \ldots, \widetilde{\theta}_{n}\right)$, respectively.

A detail explanation for the general mathematical model will be given in the following subsections in three aspects, sub-models A, B, and C.

And objective function (8) to be optimized can also be any kind of (9) to be minimized by artificial intelligence methods as follows:

$$
\begin{gathered}
F=\sum_{i=1}^{N}\left\|L_{i}-\widetilde{L}_{i}\right\|_{2}, \\
G=\frac{1}{N} \sum_{i=1}^{N}\left\|L_{i}-\widetilde{L}_{i}\right\|^{2},
\end{gathered}
$$

$$
\begin{gathered}
H=\sum_{i=1}^{N}\left\|L_{i}-\widetilde{L}_{i}\right\|^{2}, \\
W=\frac{1}{N} \sum_{i=1}^{N}\left\|L_{i}-\widetilde{L}_{i}\right\|_{2} .
\end{gathered}
$$

2.2. Mathematical Submodel A. It should be noticed here that the independent variables in function (8) in the general model in Figure 1 are not always the parameters and fractional orders. They can be the special variables, for instance, functions $\tilde{f}=\left(\tilde{f}_{1}, \tilde{f}_{2}, \ldots, \tilde{f}_{n}\right)$, fractional orders $\widetilde{q}=\left(q_{1}, q_{2}, \ldots, q_{n}\right)$, and time delays $\widetilde{\tau}=\left(\widetilde{\tau}_{1}, \widetilde{\tau}_{2}, \ldots, \widetilde{\tau}_{n}\right)$.

And for the submodel A, that is,

$$
(\widetilde{f}, \tilde{q}, \widetilde{\tau})^{*}=\underset{(\widetilde{f}, \tilde{q}, \tilde{\tau})}{\arg \min } F,
$$

it can also be written as follows:

$$
\begin{gathered}
\left(\left(\tilde{f}_{1}, \tilde{f}_{2}, \ldots, \tilde{f}_{n}\right),\left(\tilde{q}_{1}, \tilde{q}_{2}, \ldots, \tilde{q}_{n}\right),\left(\widetilde{\tau}_{1}, \tilde{\tau}_{2}, \ldots, \tilde{\tau}_{n}\right)\right)^{*} \\
=\underset{\left(\left(\tilde{f}_{1}, \tilde{f}_{2}, \ldots, \tilde{f}_{n}\right),\left(\tilde{q}_{1}, \tilde{q}_{2}, \ldots, \tilde{q}_{n}\right),\left(\tilde{\tau}_{1}, \tilde{\tau}_{2}, \ldots, \tilde{\tau}_{n}\right)\right)}{\arg \min } F .
\end{gathered}
$$

There exist several definitions of fractional derivatives. Among these, the Grünwald-Letnikov (G-L), the RiemannLiouville (R-L), and the Caputo fractional derivatives are the commonly used [40-45]. And G-L, R-L, and Caputo fractional derivatives are equivalent under some conditions [46].

The continuous integrodifferential operator $[47,48]$ is used, and we consider the continuous function $f(t)$. The G-L fractional derivatives are defined as follows:

$$
{ }_{\alpha} \mathscr{D}_{t}^{q} f(t)=\lim _{h \rightarrow 0} \frac{1}{h^{q}} \sum_{j=0}^{[(t-\alpha) / h]}(-1)^{j}\left(\begin{array}{l}
q \\
j
\end{array}\right) f(t-j h),
$$

where $[x]$ means the integer part of $x, \alpha$, and $t$ which are the bounds of operation for ${ }_{\alpha} \mathscr{D}_{t}^{q} f(t), q \in \mathfrak{R}$.

We take ideas of a numerical solution method $[47,48]$ obtained by relationship (12) derived from the G-L definition to resolve system. That is,

$$
\left(k-L_{m} / h\right) \mathscr{D}_{t_{k}}^{q} f(t) \approx \frac{1}{h^{q}} \sum_{j=0}^{k}(-1)^{j}\left(\begin{array}{l}
q \\
j
\end{array}\right) f\left(t_{k-j}\right),
$$

where $L_{m}$ is the memory length, $t_{k}=k h, h$ is the time step of calculation, and $(-1)^{j}\left(\begin{array}{c}q \\ j\end{array}\right)$ are binomial coefficients $c_{j}^{(q)}(j=0,1, \ldots)$. When, for numerical computation, the following are used:

$$
c_{0}^{(q)}=1, \quad c_{j}^{(q)}=\left(1-\frac{1+q}{j}\right) c_{j-1}^{(q)},
$$

then in general, for the simplest case (15) of (6) as following.

$$
{ }_{0} \mathscr{D}_{t}^{q} f(y(t))=f(t, y(t), y(t-\tau)) \text {. }
$$




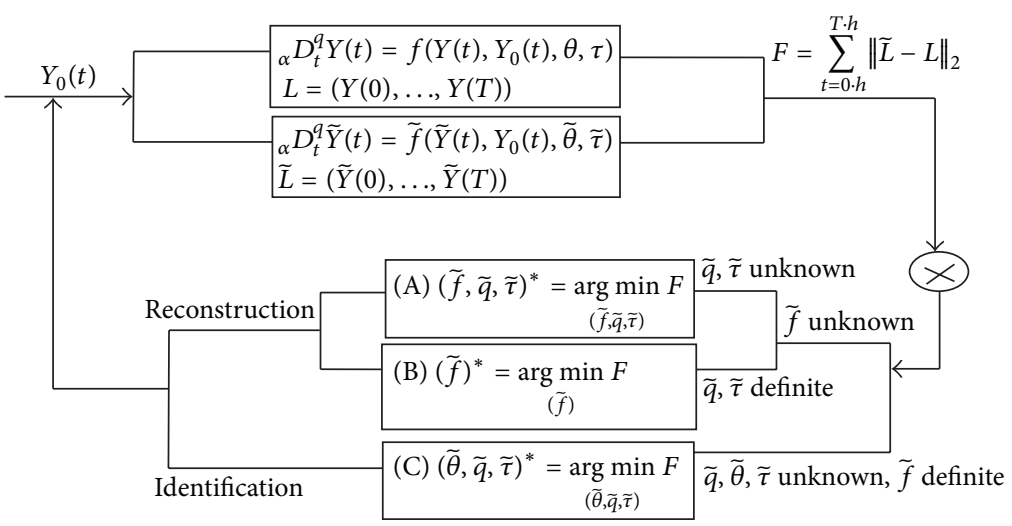

FIGURE 1: Gao's mathematical model for fractional chaos reconstruction.

Let $y_{k \tau}=y\left(t_{k}-\tau\left(t_{k}\right)\right)$. It can have the approximate value as (16), when it used for calculating

$$
\begin{aligned}
y_{k \tau}= & y_{k+1+[\tau / h]} \frac{\tau / h-[\tau / h]}{h} \\
& +y_{k+[\tau / h]} \frac{1+[\tau / h]-\tau / h}{h} .
\end{aligned}
$$

And let $y_{k}=y\left(t_{k}\right)$; then (15) can be expressed as

$$
\begin{gathered}
h^{-q} \sum_{j=0}^{k} c_{j}^{(q)} y\left(t_{k-j}\right) \\
=f\left(t_{k}, y\left(t_{k}\right), y\left(t_{k}-\tau\left(t_{k}\right)\right)\right), \\
y\left(t_{k}\right)=f\left(t_{k}, y\left(t_{k}\right), y\left(t_{k}-\tau\left(t_{k}\right)\right)\right) h^{q} \\
\quad-\sum_{j=v}^{k} c_{j}^{(q)} y\left(t_{k-j}\right), \\
y_{k}=f\left(t_{k}, y_{k}, y_{k \tau}\right) h^{q}-\sum_{j=v}^{k} c_{j}^{(q)} y_{k-j},
\end{gathered}
$$

where $v$ in the above is defined as

$$
v= \begin{cases}k-\frac{L_{m}}{h}, & k>\frac{L_{m}}{h}, \\ 1, & k<\frac{L_{m}}{h}\end{cases}
$$

or $v=1$ for all $k$.

Equation (17) is an implicit nonlinear equation respect to $y_{k}$. Now we can construct an iteration algorithm to solve $y_{k}$ as following (19).

$$
y_{k}^{(l+1)}=f\left(t_{k}, y_{k}^{(l)}, y_{k \tau}^{(l)}\right) h^{q}-\sum_{j=v}^{k} c_{j}^{(q)} y_{k-j},
$$

where $l$ is the iteration number. When $\left|y_{k}^{(l+1)}-y_{k}^{(l)}\right|<\delta$ (normally the given error $\delta<10^{-6}$ ), we consider $y_{k}=y_{k}^{(l+1)}$ to be the solution of the simplest equation (15). And if the derivative of $f$ exists $|f| \leq M$ ( $M$ is constant), and $h^{q} M<1$, then iteration (19) converges to a constant as long as the calculus step $h$ is smaller enough.

With the ideas from iteration of (19), the systems (6) and (7) are solved as follows:

$$
\begin{aligned}
& x_{i}^{(l+1)} \\
& =f_{i}\left(t_{k-1}, t_{k}, x_{1}{ }_{k}^{(l)}, x_{1}{ }_{k, \tau}^{(l)}, \ldots, x_{i-1} \stackrel{(l)}{k}, x_{i-1}{ }_{k, \tau}^{(l)},\right. \\
& \left.x_{i}{ }_{k-1}^{(l)}, x_{i}{ }_{k-1, \tau}^{(l)}, \ldots, x_{n-1}^{(l)}, x_{n-1, \tau}^{(l)}\right) h^{q_{i}} \\
& -\sum_{j=v}^{k} c_{j}^{(q)} x_{i k-j}, \\
& \tilde{x}_{i k}^{(l+1)} \\
& =\tilde{f}_{i}\left(t_{k-1}, t_{k}, \tilde{x}_{1 k}^{(l)}, \tilde{x}_{1 k, \tilde{\tau}}^{(l)}, \ldots, \tilde{x}_{i-1} \stackrel{(l)}{k}, \tilde{x}_{i-1} \stackrel{(l)}{k, \widetilde{\tau}},\right. \\
& \left.\tilde{x}_{i k-1}^{(l)}, \tilde{x}_{i k-1, \tilde{\tau}}^{(l)}, \ldots, \tilde{x}_{n k-1}^{(l)}, \tilde{x}_{n k-1, \tilde{\tau}}^{(l)}\right) h^{\widetilde{q}_{i}} \\
& -\sum_{j=v}^{k} c_{j}^{(\widetilde{q})} \tilde{x}_{i k-j} .
\end{aligned}
$$

And if $\tau=(0,0, \ldots, 0)$, then the system (6) can be solved as [36]

$$
\begin{aligned}
x_{i k}^{(l+1)} & \\
= & f_{i}\left(x_{1}{ }_{k}^{(l)}, \ldots, x_{i-1}{ }_{k}^{(l)}, x_{i}{ }_{k-1}^{(l)}, \ldots, x_{n k-1}{ }_{k}^{(l)}\right) h^{q_{i}} \\
& \quad-\sum_{j=v}^{k} c_{j}^{\left(q_{i}\right)} x_{i k-j} .
\end{aligned}
$$

To the best of our knowledge, there is no work that has been done to reconstruct the fractional chaos systems under condition that both $f, q$ and $\tau$ are unknown in submodel A as (10) neither for time-delays free nor with time-delays chaos systems. 
2.3. Mathematical Submodel B. In this sub-model, $f$ are unknown, but $\tau$ and $q$ are definite. Then to be estimated is only the fractional differential equations $f$; that is,

$$
(\tilde{f})^{*}=\arg \min F .
$$

It is should be noticed that there are few methods for reconstruction of fractional-order chaos systems [36] so far.

However, there are a few results for normal chaos systems, as the special cases of fractional chaos systems. For reconstruction of $f=\left(f_{1}, f_{2}, \ldots, f_{n}\right)$ with the non-Lyapunov methods, they are mainly from symbolic regression through genetic programming (GP) [49-51], and some are from evolutionary algorithms [52-62].

Considering mathematical sub-model $\mathrm{A}$, we have to say that it is really difficult to use the ideas in mathematical sub-model B. Let the input variables be taken as $x, y$, and $z$ and let the basic operators set used be $\left\{+,-, \times, \div, \mathscr{D}_{t}^{p},=\right.$ \} , where fractional-order $p \in[0,1]$ is uncertain. Now we consider the easiest cases that the fractional order differential equation $\mathscr{D}_{t}^{q_{1}} x=f_{1}(x, y, z)$ is unknown. Then we will see the individuals as following with the ideas similar to methods for the normal chaos of sub-model B. Figures 2(a) and 2(b) are the normal and correct candidate individuals only for the right part $f_{1}$ of $d x / d t=f_{1}(x, y, z)$ of the normal chaos systems.

However, when it comes to fractional-order chaos system, the whole fractional-order differential equations should be taken into accounts; that is, $\mathscr{D}_{t}^{q_{1}} x=f_{1}(x, y, z)$ with $q=$ $p \in[0,1]$ uncertain and $f_{1}$ unknown. Figure 2(c) shows a correct candidate. And when the evolutions (crossover, mutation, and selection) go on, there are some wrong and illegal candidates generated as Figures 2(d), 2(e), and 2(f) show. Figure $2(\mathrm{~d})$ is a wrong candidate with $\mathscr{D}_{t}^{p}(x-y)$. Figure 2(e) is a wrong candidate with $\mathscr{D}_{t}^{p_{1}}(x-y)$ and $\mathscr{D}_{t}^{p_{2}}(y)$. Here it should be noticed that random $p_{1}, p_{2} \in[0,1]$. Figure $2(\mathrm{f})$ is a wrong candidate with not only $\mathscr{D}_{t}^{p_{1}}(x-y)$, $\mathscr{D}_{t}^{p_{2}}(z)$, and $\mathscr{D}_{t}^{p_{3}}(z)$ but also extra $\{=\}$. Here it should be noticed that random $p_{1}, p_{2}, p_{3} \in[0,1]$.

So long as the evolutions (crossover, mutation, and selection) go on, these wrong candidate inevitably exists, although, in the genetic programming, the tree depth is set to be limited with unlimited leaves. And these kinds of wrong individuals will become heavy burden for both the genetic evolution and resolving of the fractional-order differential equations.

Thus it is not suitable to use the methods based on GP only to reconstruct the fractional-order chaos system; neither fractional order $q$ nor equations $f_{i}$ are unknown. However, if it is only considering the unknown equations $f_{i}$ with definite certain fractional order $q$, these methods will be impressive and efficient as in [36].

2.4. Mathematical Submodel C. In this sub-model C, $\left(q_{1} \neq q_{2} \neq \cdots \neq q_{n}\right)$, systematic parameters and time delays $\left(\theta_{1}, \theta_{2}, \ldots, \theta_{n}\right), \widetilde{\tau}=\left(\widetilde{\tau}_{1}, \widetilde{\tau}_{2}, \ldots, \widetilde{\tau}_{n}\right) \in\left(f_{1}, f_{2}, \ldots, f_{n}\right)$ are unknown for noncommensurate and hyperfractional-order chaos system.
There are some estimation methods that have been purposed to identify the unknown parameters and orders for commensurate fractional-order chaotic systems. However, to the best of our knowledge, no such reconstruction methods have been done for noncommensurate and hyper fractionalorder chaos system; it is necessary to resolve the following equation in non-Lyapunov way:

$$
(\tilde{q}, \tilde{\theta}, \tilde{\tau})^{*}=\arg \min _{(\tilde{q}, \tilde{\theta}, \tilde{\tau})} F .
$$

And there exist basic hypotheses in traditional nonLyapunov estimation methods for fractional-order systems $[7,9,28]$. That is, the parameters and fractional orders are partially known or the known data series coincide with definite forms $f=\left(f_{1}, f_{2}, \ldots, f_{n}\right)$ of fractional chaotic differential equations except for some uncertain parameters and fractional orders $\Theta=\left(\theta_{1}, \theta_{2}, \ldots, \theta_{n}, q_{1}, q_{2}, \ldots, q_{n}\right)$.

This is the basic difference between submodel A, B, and C. And for the case when some chaotic differential equations $f=\left(f_{1}, f_{2}, \ldots, f_{n}\right)$ are unknown, there are some chaos system reconstruction methods. Then the cases [6375] can be thought of as special cases of chaos reconstruction, when the exact forms of chaotic differential equations $f=\left(f_{1}, f_{2}, \ldots, f_{n}\right)$ are available, but some parameters are unknown.

2.4.1. Parameters Estimation for Fractional-Order Chaos Systems. We take the fractional-order Lorénz system (25) [3, $8,24]$ as an example, which is generalized from the first canonical chaotic attractor found in 1963, Lorénz system [76]:

$$
\begin{gathered}
{ }_{\alpha} \mathscr{D}_{t}^{q_{1}} x=\sigma \cdot(y-x), \\
{ }_{\alpha} \mathscr{D}_{t}^{q_{2}} y=\gamma \cdot x-x \cdot z-y, \\
{ }_{\alpha} \mathscr{D}_{t}^{q_{3}} z=x \cdot y-b \cdot z, \\
L=(x, y, z),
\end{gathered}
$$

where $q_{1}, q_{2}$, and $q_{3}$ are the fractional orders. When $\left(q_{1}, q_{2}, q_{3}\right)=(0.993,0.993,0.993), \sigma=10, \gamma=28, b=8 / 3$, and $\alpha=0$, intimal point $(0.1,0.1,0.1)$ system (25) is chaotic. Generally when the dimension

$$
\sum=q_{1}+q_{2}+q_{3}>2.91
$$

for fractional system $(25)$ is chaotic $[3,8,24]$, the form of function (10) can also be as follows:

$$
p^{2}=F\left(\sigma, \gamma, b, q_{1}, q_{2}, q_{3}\right)=\sum_{t=0 \cdot h}^{T \cdot h}\|\widetilde{L}-L\|^{2} .
$$

It is noticed that objective function (27) can be any forms of correspond equations (9).

Then the problems of estimation of parameters for chaotic system are transformed into those of nonlinear function optimization (27). And the smaller the $p^{2}$ is, the better are combinations of parameter $\left(\sigma, \gamma, b, q_{1}, q_{2}, q_{3}\right)$. The independent variables of these functions are $\theta=\left(\sigma, \gamma, b, q_{1}, q_{2}, q_{3}\right)$. 


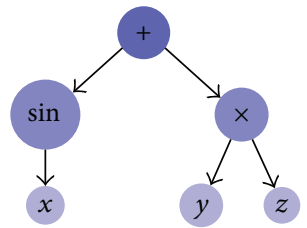

(a) $\sin x+y z$

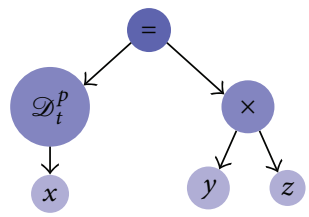

(c) $\mathscr{D}_{t}^{p} x=y z$

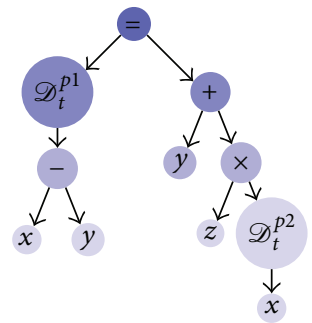

(e) $\mathscr{D}_{t}^{p_{1}}(x-y)=y+$ $z \mathscr{D}_{t}^{p_{2}} x$

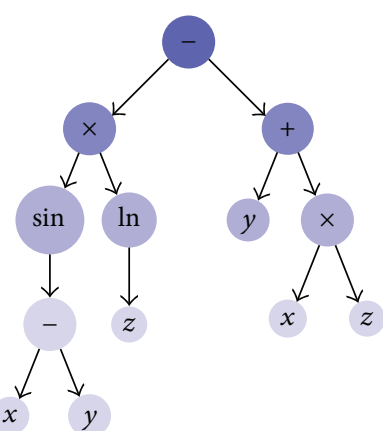

(b) $\sin (x-y) \cdot \ln z-(y+x z)$

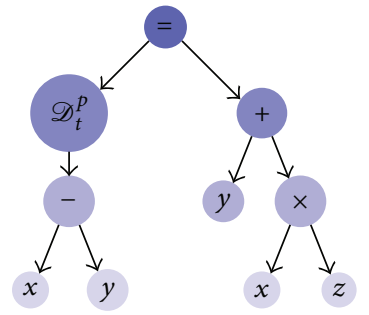

(d) $\mathscr{D}_{t}^{p}(x-y)=y+x z$

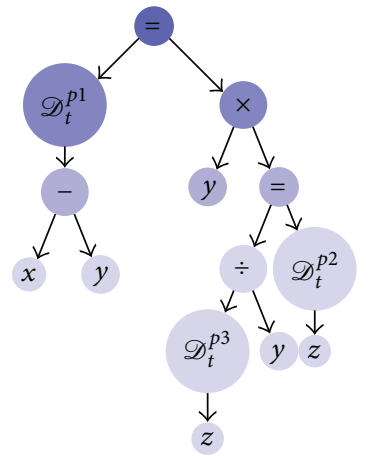

(f) Illegal candidates

FIGURE 2: Some examples of the tree structures in GP evolutions.

And considering that the fractional system is very complicated, to simplify the problems, it is reported unknown $q=q_{1}=q_{2}=q_{3}, \sigma, \gamma, b$ or case of $\sigma, \gamma, b$ are known and only one $q_{i}$ are unknown for the similar fractional-order chaos systems, such as fractional order Lü system [16, 77], fractional-order Chen system [27, 78], and fractional Lorénz system $[3,8,24]$, discussed in $[7,9]$. This is the basic idea for the recently proposed methods for fractional chaos system $[7,9]$.

However, the case $q_{1} \neq q_{2} \neq q_{3}$ is not included in the above non-Lyapunov ideas or not fully discussed either for noncommensurate fractional chaos systems.

2.5. The Main Differences between Submodels A, B, and $C$. Equation (10) is the crucial turning point that changing from the parameters estimation into functions reconstruction and orders estimation, in other words, both fractional-order estimation and fractional chaos systems' reconstruction.
It can be concluded that the parameters' estimation of fractional-order chaos system $[7,9]$ is a special case of fractional-order chaos reconstruction here as (10). In their researches, the forms of the fractional-order differential equations $\left(f_{1}, f_{2}, \ldots, f_{n}\right)$ are known, but some parameters $\left(\theta_{1}, \theta_{2}, \ldots, \theta_{n}\right)$ of these equations are unknown, and only one fractional order and some of these systematic parameters $\left(\theta_{1}, \theta_{2}, \ldots, \theta_{n}\right)$ are estimated $[7,9]$.

And further, the parameters estimation cases that all $\left(f_{1}, f_{2}, \ldots, f_{n}\right)$ are known but parameters $\left(\theta_{1}, \theta_{2}, \ldots, \theta_{n}\right)$ of these equations are unknown and the reconstruction case that some of $\left(f_{1}, f_{2}, \ldots, f_{n}\right)$ are unknown, in Section 2.3 for the normal chaos system, are the special cases of fractional-order chaos systems' reconstruction (10).

However, it should be emphasized here that, for reconstruction the novel general mathematical model (10) for fractional chaos parameters identification in non-Lyapunov way, with uncertain different fractional order $q$; that is, $q_{1} \neq q_{2} \neq \cdots \neq q_{n} \in\left\{\times, \div,+,-, \mathscr{D}_{t}^{q_{1}}, \mathscr{D}_{t}^{q_{2}}, \ldots, \mathscr{D}_{t}^{q_{n}}\right\} ;$ it is really 
difficulty to generate a proper candidate from this basic set as shown in Figure 2. Then, it is not easy to reconstruct the fractional-order differential equations and identify the fractional orders together. And only the simplest case that with definite $q=q_{1}=q_{2}=\cdots=q_{n}$ is discussed [36].

\section{Cuckoo Search Oriented Statistically by Differential Evolution}

3.1. Cuckoo Search. Cuckoo search (CS) is an optimization algorithm [37, 38], inspired by the obligate brood parasitism of some cuckoo species by laying their eggs in the nests of other host birds (of other species). And some host birds can come into direct conflict with the intruding cuckoos.

CS is based on three idealized rules.

(i) Only one egg is laid and is dumped into a randomly chosen nest by each cuckoo at time $t$.

(ii) The best nests with high quality of eggs (candidate solutions) will be copied to the next generation directly.

(iii) The number of available host nests is fixed, and an alien egg will be discovered by a host bird with probability $p_{a} \in[0,1]$. If so, the host can either throw the egg away or abandon the nest so as to build a completely new nest in a new location.

A Lévy flight is performed for cuckoo $i$ when a new candidate $x^{(t+1)}$ is generated [37-39]:

$$
x_{i}^{(t+1)}=x_{i}^{(t)}+a \oplus \operatorname{Lévy}(\lambda),
$$

where $a>0$ is the step size which should be related to the scales of the problem of interest. Normally, $a=O(1)$. The product $\oplus$ means entrywise multiplications. Lévy flights essentially provide a random walk while their random steps are drawn from a Lévy distribution for large steps

$$
\text { Lévy } \sim u=t^{-\lambda} \quad(1<\lambda \leq 3),
$$

which has an infinite variance with an infinite mean, and essentially form a random walk process obeying a power-law step-length distribution with a heavy tail [37-39].

Based on the above rules and ideas, the basic steps of the CS can be summarised as shown in pseudocode of Algorithm 1.

It should be noticed that, in each iteration of Algorithm 1, there are two rounds of evaluation of the fitness: one is after getting a cuckoo by Lévy flights and the other is after abandon the worse nests with probability $p_{a}$ and building the new nest at the new locations. It is also showed in the original MATLAB code in [38].

This might be the reason that CS is efficient. Because CS uses Lévy flights twice and evaluates the candidates twice in one generation. However, there is one evaluation for the whole population in normal swarm intelligent methods. If we consider the number of evaluating the fitness function by these two evaluations, they might not be economic.

Thus, we can make some modifications here to accelerate the CS as Algorithm 1 by decreasing the evaluation number for the fitness.
3.2. Differential Evolution Algorithm. Differential evolution (DE) algorithm was proposed by Storn et al. [79-82]. DE utilizes $M n$-dimensional vectors, $X_{i}=\left(x_{i 1}, \ldots, x_{i n}\right) \in$ $S, i=1, \ldots, M$, as a population for each iteration, called a generation, of the algorithm. For each vector $X_{i}^{(G)}=$ $\left(X_{i 1}^{(G)}, X_{i 2}^{(G)}, \ldots, X_{i n}^{(G)}\right), i=1,2, \ldots, M$, there are three main genetic operators acting [79-82].

To apply the mutation operator, firstly choose randomly four mutually different individuals in the current population $X_{r_{1}}^{(G)}, X_{r_{2}}^{(G)}, X_{r_{3}}^{(G)}, X_{r_{4}}^{(G)}\left(r_{1} \neq r_{2} \neq r_{3} \neq r_{4} \neq i\right)$ to compose a differential vector $D^{(G)}=\left[X_{r_{1}}^{(G)}-X_{r_{2}}^{(G)}\right]+\left[X_{r_{3}}^{(G)}-X_{r_{4}}^{(G)}\right]$; then combines it with the current best individual $X_{\text {best }}^{(G)}$ to get a perturbed vector $V=\left(V_{1}, V_{2}, \ldots, V_{n}\right)[79,83]$ as follows:

$$
V=X_{\text {best }}^{(G)}+\mathrm{CF} \times D^{(G)}
$$

where $\mathrm{CF}>0$ is a user-defined real parameter, called mutation constant, which controls the amplification of the difference between two individuals to avoid search stagnation.

Following the crossover phase, the crossover operator is applied on $X_{i}^{(G)}$. Then a trial vector $U=\left(U_{1}, U_{2}, \ldots, U_{n}\right)$ is generated by

$$
U_{m}= \begin{cases}V_{m}, & \text { if }(\text { rand }(0,1)<\mathrm{CR}) \text { or }(m=k), \\ X_{i m}^{(G)}, & \text { if }(\operatorname{rand}(0,1) \geq \mathrm{CR}) \text { and }(m \neq k),\end{cases}
$$

in the current population [79], where $m=1,2, \ldots, n$, the index $k \in\{1,2, \ldots, n\}$ is randomly chosen, and CR is a userdefined crossover constant $[79,83]$ in the range $[0,1]$. In other words, the trial vector consists of some of the components of the mutant vector and at least one of the components of a randomly selected individual of the population.

Then it comes to the replacement phase. To maintain the population size, we have to compare the fitness of $U$ and $X_{i}^{(G)}$ and then choose the better:

$$
X_{i}^{(G+1)}= \begin{cases}U, & \text { if } F(U)<F\left(X_{i}^{(G)}\right) \\ X_{i}^{(G)}, & \text { otherwise. }\end{cases}
$$

3.3. Cuckoo Search Oriented Statistically by Differential Evolution. Considering the redundant evaluation for the fitness function of CS and the efficiency of DE, we can propose a novel cuckoo search oriented statistically by differential evolution as shown in Algorithm 2.

In each iteration of Algorithm 2, Lévy flights (28) are used once for each location. And differential evolution operation is used with a probability $p_{\text {De }}$ less than 0.2 . In this way, the evaluations for the fitness function are reduced nearly by $80 \%$ compared to original Algorithm 1.

And $p_{D e}$ in Algorithm 2 CSODE should not be too big. Otherwise, it will cause Algorithm 2 to be much more like a DE algorithm rather than a cuckoo searcher algorithm. It will be illustrated in the section of simulations. Actually, our original idea is to let CS be oriented not controlled by DE. 
(1) Basic parameters' setting Objective function $f(x), x=\left(x_{1}, \ldots, x_{d}\right)$, initial population of $n$ host nests $x_{i}(i=1,2, \ldots, n)$, boundaries for each dimension $x_{i}=\left(x_{i, 1}, \ldots, x_{i, d}\right)$ and so forth.

(2) While Termination condition is not satisfied do

(3) Get a cuckoo $(i, i=1,2, \ldots, n)$ randomly by Lévy flights (28).

(4) Evaluate its fitness $F_{i}$;

(5) If $\left(F_{i}>F_{j}\right)$

(6) Replace $j$ by the new solution

(7) End

(8) Abandon a fraction $\left(p_{a}\right)$ of worse nests.

(9) Build new ones at new locations via Lévy flights (28) $(i, i=1,2, \ldots, n)$.

(10) Keep the best solutions (or nests with quality solutions).

(11) Rank the solutions and find the current best.

(12) end while

(13) Output Global optimum $Q_{g}$

Algorithm 1: The basic steps of the cuckoo search.

(1) Basic parameters' setting Objective function $f(x), x=\left(x_{1}, \ldots, x_{d}\right)$, initial population of $n$ host nests $x_{i}(i=1,2, \ldots, n)$, boundaries for each dimension $x_{i}=\left(x_{i, 1}, \ldots, x_{i, d}\right)$ and so forth.

(2) While Termination condition is not satisfied do

(3) If $p_{\mathrm{De}}<0.2$, generating candidate cuckoo population $(i, i=1,2, \ldots, n)$ randomly from current population by (31).

(4) Updating the current cuckoo swarm and the candidate swarm with (32).

(5) Abandon a fraction $\left(p_{a}\right)$ of worse nests.

(6) Build new ones at new locations via Lévy flights (28).

(7) Keep the best solutions (or nests with quality solutions).

(8) Rank the solutions and find the current best.

(9) end while

(10) Output Global optimum $Q_{g}$

Algorithm 2: Cuckoo search oriented statistically by differential evolution (CSODE).

\section{A Novel Unknown Parameters and Orders Identification Method Based on CSODE for Noncommensurate Fractional-Order Chaos Systems}

The task of this section is to find a simple but effective approach for unknown $q$ and systematic parameters in (24) for noncommensurate fractional-order chaos based on CSODE in non-Lyapunov way.

4.1. A Novel Unknown Parameters and Orders Identification Method. Now we can propose a novel approach for hyper, proper, and improper fractional chaos systems. The pseudocode of the proposed reconstruction is given in Algorithm 3.

4.2. Noncommensurate and Hyperfractional-Order Chaos Systems. To test Algorithm 3, some different well-known and widely used noncommensurate and hyper fractional order chaos systems are choose as follows. To have a comparative result, these systems are taken from [36].
Example 1. Here we discuss the noncommensurate fractional Lorénz system $[8,36]$.

Example 2. Fractional-order Arneodo's system [36, 47, 84].

Example 3. Fractional-order Duffing's system [47].

Example 4. Fractional-order Genesio-Tesi’s systems [47, 85].

Example 5. Fractional-order financial systems [36, 47, 85].

Example 6. Fractional-order Lü system $[16,47]$.

Example 7. Improper fractional-order Chen system [27, 47, 78].

Example 8. Fractional-order Rössler system [12, 47].

Example 9. Fractional-order Chua's oscillator [86].

Example 10. Hyperfractional-order Lorénz system [87].

Example 11. Hyperfractional-order Lü system [88]. 


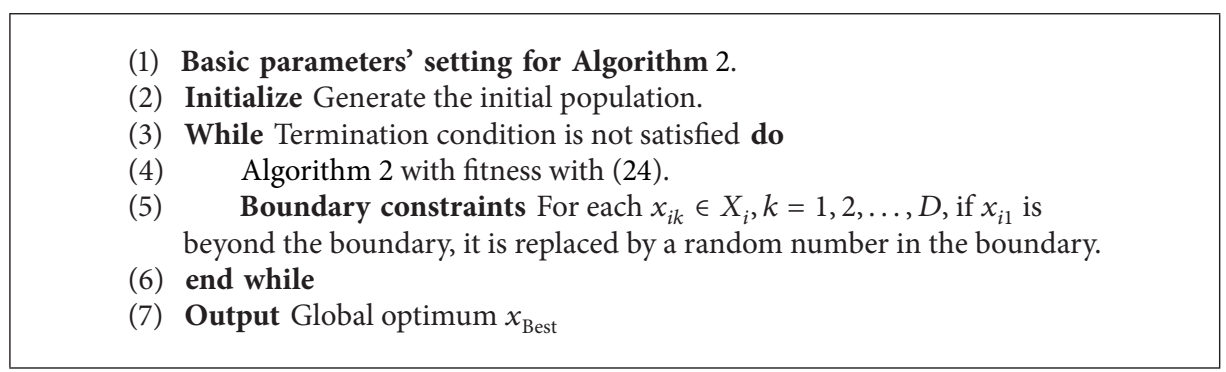

Algorithm 3: A novel unknown parameters and orders identification method based on differential evolution algorithms for noncommensurate and hyperfractional-order chaos systems.

TABLE 1: Detail parameters setting for different systems.

\begin{tabular}{lccccc}
\hline F-O systems & Unknown & Lower boundary & Upper boundary & Step $^{\text {NOSP }}$ \\
\hline Example 1 & $\left(\sigma, \gamma, b, q_{1}, q_{2}, q_{3}\right)$ & $5,20,0.1,0.1,0.1,0.1$ & $15,30,10,1,1,1$ & 0.01 & 100 \\
Example 2 & $\left(\beta_{1}, \beta_{2}, \beta_{3}, \beta_{4}, q_{1}, q_{2}, q_{3}\right)$ & $-6,2,0.1,-1.5,0.1,0.1,0.1$ & $-5,5,1,-0.5,1,1,1$ & 0.005 & 200 \\
Example 3 & $\left(a, b, c, q_{1}, q_{2}\right)$ & $0.1,0.1,0.1,0.1,0.5$ & $1,1,2,1,1.5$ & 0.0005 & 500 \\
Example 4 & $\left(\beta_{1}, \beta_{2}, \beta_{3}, \beta_{4}, q_{1}, q_{2}, q_{3}\right)$ & $1,1,0.1,0.1,0.5,0.5,0.1$ & $2,2,1,1.5,1.5,1.5,1$ & 0.005 & 200 \\
Example 5 & $\left(a, b, c, q_{1}, q_{2}, q_{3}\right)$ & $0.5,0.01,0.5,0.5,0.1,0.1$ & $1.5,1,1.5,1.5,1,1$ & 0.005 & 200 \\
Example 6 & $\left(a, b, c, q_{1}, q_{2}, q_{3}\right)$ & $30,0.1,15,0.1,0.1,0.1$ & $40,10,25,1,1,1$ & 0.01 & 100 \\
Example 7 & $\left(a, b, c, d, q_{1}, q_{2}, q_{3}\right)$ & $30,0.1,20,-10,0.5,1,1$ & $40,10,30,-0.1,2,2,2$ & 0.01 & 100 \\
Example 8 & $\left(a, b, c, q_{1}, q_{2}, q_{3}\right)$ & $0.1,0.1,5,0.1,0.1,0.1$ & $1,1,15,1,1,1$ & 0.01 & 100 \\
Example 9 & $\left(\alpha, \beta, \zeta, a, b, q_{1}, q_{2}, q_{3}, q_{4}\right)$ & $5,10,0.1,0.1,0.3,0.1,0.1,0.1,0.1,0.1$ & $10,20,1,2,0.3,1,1,1,1,1$ & 0.01 & 100 \\
Example 10 & $\left(a, b, c, d, q_{1}, q_{2}, q_{3}, q_{4}\right)$ & $5,0.1,20,-2,0.1,0.1,0.1,0.1$ & $15,5,30,-0.1,1,1,1,1$ & 0.01 & 100 \\
Example 11 & $\left(a, b, c, d, q_{1}, q_{2}, q_{3}, q_{4}\right)$ & $30,0.1,15,0.1,0.1,0.1,0.1,0.1$ & $40,5,25,5,1,1,1,1$ & 0.005 & 200 \\
Example 12 & $\left(a, b, c, d, q_{1}, q_{2}, q_{3}, q_{4}\right)$ & $5,0.5,1,0.1,0.1,0.1,0.1,0.1$ & $15,1.5,10,1,1,1,1,1$ & 0.005 & 100 \\
Example 13 & $\left(a, b, c, d, q_{1}, q_{2}, q_{3}, q_{4}\right)$ & $30,0.1,10,0.1,0.1,0.1,0.1,0.1,0.1$ & $40,5,20,10,1,1,1,1,1$ & 0.005 & 200 \\
Example 14 & $\left(a, b, c, d, q_{1}, q_{2}, q_{3}, q_{4}\right)$ & $0.1,0.1,0.1,0.01,0.1,0.1,0.1,0.1$ & $1,5,1,1,1,1,1,1$ & 0.005 & 200 \\
Example 15 & $\left(a, b, c, q_{1}, q_{2}, q_{3}, q_{4}\right)$ & $5,38,45,0.5,0.5,0.5,0.5$ & $10,45,50,1,1,1,1$ & 0.005 & 200 \\
\hline
\end{tabular}

${ }^{\mathrm{a}}$ Number of sample points for resolving the fractional chaos systems.

Example 12. Hyperfractional-order Liu system [89].

Example 13. Hyperfractional-order Chen system [90].

Example 14. Hyperfractional-order Rössler system [12].

Example 15. A four-wing fractional-order system [91, 92] both incommensurate and hyperchaotic

$$
\begin{gathered}
\mathscr{D}_{t}^{q_{1}} x_{1}=a x_{1}-x_{2} x_{3}+x_{4}, \\
\mathscr{D}_{t}^{q_{2}} x_{2}=-b x_{2}+x_{1} x_{3}, \\
\mathscr{D}_{t}^{q_{3}} x_{3}=x_{1} x_{2}-c x_{3}+x_{1} x_{4}, \\
\mathscr{D}_{t}^{q_{4}} x_{4}=-x_{2} .
\end{gathered}
$$

When $(a, b, c)=(8,40,49),\left(q_{1}, q_{2}, q_{3}, q_{4}\right)=(1,0.95,0.9$, $0.85)$, and initial point is $(1,-2,3,1)$ [91], system (33) is chaotic.

4.3. Simulations. For systems to be identified, the parameters of the proposed method are set as follows. The parameters of the simulations are fixed: the size of the population was set equal to $M=40$, generation is set to 500 , and the default values are $\mathrm{CF}=1, \mathrm{CR}=0.85$, and $p_{\mathrm{DE}}=0.2$; Table 1 gives the detail setting for each system.

Table 2 shows the simulation results of the above fractional-order chaotic systems. And some simulations are done by single cuckoo search (CS) methods. In these cases, all the other parameters for the algorithms are the same as for CSODE. The simulation results are listed in Table 3.

And comparisons of CSODE with evolutionary algorithms such as Genetic Algorithms are done. Here we choose the GA toolbox from MATLAB 2013a; most of the parameters are chosen as default in Matlab, except that population size is 80 , generation number is 600 , exiting the GA's evolution with average fitness value changes less than $10^{-16}$, and "Vectorized" is "on," "UseParallel" is "always." And simulation results are showed in Table 4.

The following figures give an illustration of how the self growing evolution process works by CSODE (Algorithm 3 ). In which, Figures $3,4,5,6,7,8,9,10$, and 11 show the simulation evolution results of the above fractional order chaotic systems with optimization process of objective function's evolution and the parameters and orders uncertain of the above fractional order chaotic systems. 
TABLE 2: Simulation results for different fractional-order chaos systems by CSODE.

\begin{tabular}{|c|c|c|c|c|c|c|}
\hline F-O system & StD & Mean & Min. & Max. & Success rate ${ }^{\mathrm{a}}$ & $\mathrm{NEOF}^{\mathrm{b}}$ \\
\hline Example 1 & $2.2025 e-04$ & $6.6862 e-04$ & $3.1179 e-06$ & $5.5594 e-03$ & $100 \%$ & 24013 \\
\hline Example 2 & $4.7346 e-06$ & $1.5061 e-05$ & $3.2217 e-07$ & $1.4355 e-04$ & $100 \%$ & 24073 \\
\hline Example 3 & $5.8073 e-09$ & $1.4890 e-08$ & $1.3035 e-10$ & $1.3548 e-07$ & $100 \%$ & 24016 \\
\hline Example 4 & $1.3724 e-04$ & $5.1929 e-04$ & $7.6119 e-07$ & $3.8262 e-03$ & $100 \%$ & 24073 \\
\hline Example 5 & $2.7967 e-08$ & $2.9039 e-08$ & $1.5084 e-09$ & $1.4728 e-07$ & $100 \%$ & 24013 \\
\hline Example 6 & $2.7950 e-04$ & $6.5471 e-04$ & $6.8653 e-06$ & $5.9367 e-03$ & $100 \%$ & 24038 \\
\hline Example 7 & $6.5650 e-03$ & $8.3188 e-03$ & $1.6981 e-04$ & $4.3289 e-02$ & $83 \%$ & 24074 \\
\hline Example 8 & $1.8666 e-08$ & $2.1368 e-08$ & $1.2494 e-09$ & $1.2324 e-07$ & $100 \%$ & 24013 \\
\hline Example 9 & $1.4857 e-04$ & $1.4529 e-04$ & $1.9011 e-05$ & $1.0265 e-03$ & $100 \%$ & 24073 \\
\hline Example 10 & $5.1741 e-03$ & $4.4624 e-03$ & $5.0835 e-04$ & $2.4994 e-02$ & $87 \%$ & 24041 \\
\hline Example 11 & $1.2904 e-02$ & $1.2573 e-02$ & $5.8260 e-04$ & $5.5329 e-02$ & $100 \%^{\mathrm{c}}$ & 24041 \\
\hline Example 12 & $6.2490 e-06$ & $4.1206 e-06$ & $3.3623 e-07$ & $2.3320 e-05$ & $100 \%$ & 24101 \\
\hline Example 13 & $5.4440 e-02$ & $4.5710 e-02$ & $6.0972 e-03$ & $2.7476 e-01$ & $87 \%^{\mathrm{c}}$ & 24022 \\
\hline Example 14 & $2.0859 e-04$ & $1.5394 e-04$ & $3.1938 e-05$ & $7.9808 e-04$ & $100 \%$ & 24041 \\
\hline Example 15 & $4.5612 e-01$ & $1.8007 e+00$ & $3.1952 e-03$ & $1.6640 e+01$ & $56 \%^{\mathrm{c}}$ & 24024 \\
\hline
\end{tabular}

${ }^{\mathrm{a}}$ Success means that the solution is less than $1 e-2$ in 100 independent simulations.

${ }^{\mathrm{b}}$ No. of average evaluation for objective function (NEOF).

${ }^{c}$ Success means that the solution is less than $1 e-1$ in 100 independent simulations.

TABLE 3: Simulation for fractional-order systems by single cuckoo search.

\begin{tabular}{|c|c|c|c|c|c|c|}
\hline System & $\mathrm{StD}$ & Mean & Min. & Max. & Success rate ${ }^{\mathrm{a}}$ & $\mathrm{NEOF}^{\mathrm{b}}$ \\
\hline Example 1 & $3.8566 e-01$ & $6.8548 e-01$ & $1.1083 e-01$ & 2.2250 & $0 \%$ & 40040 \\
\hline Example 2 & $5.2993 e-04$ & $1.1322 e-03$ & $2.9726 e-04$ & $2.7731 e-03$ & $100 \%$ & 40040 \\
\hline Example 3 & $2.2560 e-04$ & $3.8504 e-04$ & $7.2187 e-05$ & $1.4730 e-03$ & $100 \%$ & 40040 \\
\hline Example 4 & $4.0392 e-03$ & $1.8645 e-03$ & $1.0365 e-03$ & $1.0422 e-02$ & $100 \%$ & 40040 \\
\hline Example 6 & $9.2611 e-01$ & 1.8303 & $6.0910 e-01$ & 6.4697 & $0 \%$ & 40040 \\
\hline Example 9 & $8.1825 e-02$ & $4.0495 e-02$ & $1.5058 e-02$ & $2.3472 e-01$ & $0 \%$ & 40040 \\
\hline Example 10 & 9.5917 & 4.4177 & 3.1812 & $2.5456 e+01$ & $0 \%$ & 40040 \\
\hline Example 11 & 3.8130 & 7.1730 & 2.5631 & 2.7080 & $0 \%$ & 40040 \\
\hline Example 13 & 8.8389 & 3.5136 & 2.6274 & $2.4436 e+01$ & $0 \%$ & 40040 \\
\hline Example 15 & $9.5876 e-02$ & $1.4075 e-01$ & $3.3331 e-02$ & $5.7142 e-01$ & $43 \%$ & 40040 \\
\hline
\end{tabular}

${ }^{a}$ Success means that the solution is less than $1 e-1$ in 100 independent simulations.

${ }^{\mathrm{b}}$ Number of evaluation for objective function.

TABLE 4: Simulation for fractional-order systems by Genetic Algorithm.

\begin{tabular}{|c|c|c|c|c|c|c|}
\hline System & StD & Mean & Min. & Max. & Success rate ${ }^{\mathrm{a}}$ & $\mathrm{NEOF}^{\mathrm{b}}$ \\
\hline Example 1 & 8.8774 & 7.6047 & 1.4454 & 17.78 & $0 \%$ & 48080 \\
\hline Example 2 & 0.098989 & 0.076223 & 0.0057602 & 0.1894 & $37 \%$ & 48080 \\
\hline Example 5 & 0.0074472 & 0.042908 & 0.035629 & 0.050513 & $100 \%$ & 48080 \\
\hline Example 6 & 0.52621 & 5.6488 & 5.0984 & 6.1469 & $0 \%$ & 48080 \\
\hline Example 8 & 0.025535 & 0.036555 & 0.007422 & 0.055056 & $40 \%$ & 48080 \\
\hline Example 10 & 5.5344 & 36.469 & 30.181 & 40.602 & $0 \%$ & 48080 \\
\hline Example 11 & 14.492 & 17.303 & 5.2875 & 33.397 & $0 \%$ & 48080 \\
\hline Example 13 & 4.8245 & 10.34 & 4.773 & 13.299 & $0 \%$ & 48080 \\
\hline Example 15 & 6.3815 & 721.09 & 713.72 & 724.77 & $0 \%$ & 34294 \\
\hline
\end{tabular}

${ }^{\mathrm{a}}$ Success means that the solution is less than $1 e-1$ in 100 independent simulations.

${ }^{\mathrm{b}}$ No. of evaluation for objective function. 


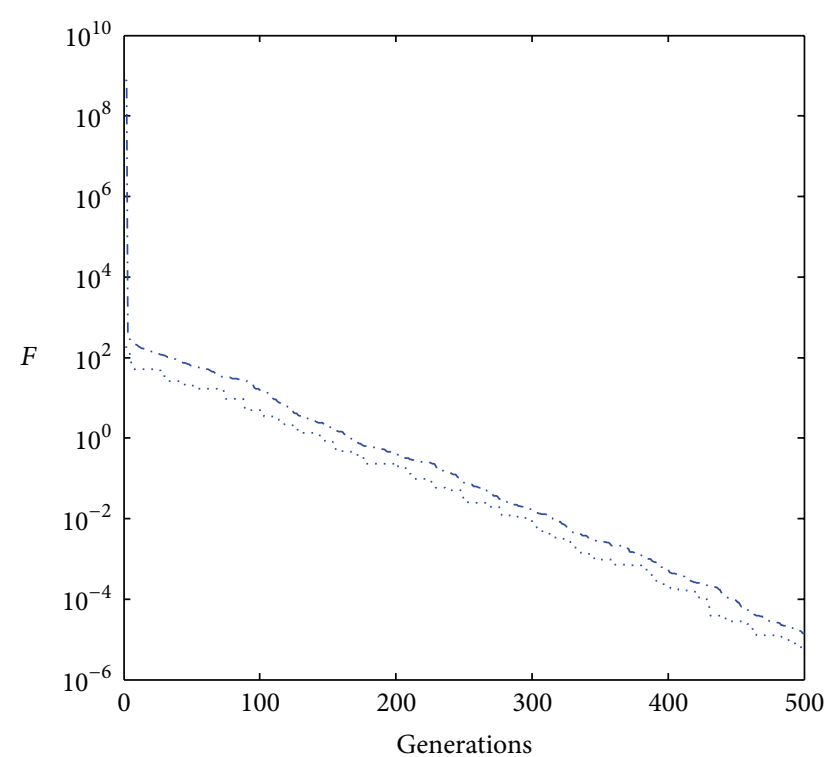

Best CSODE

-. - Mean CSODE

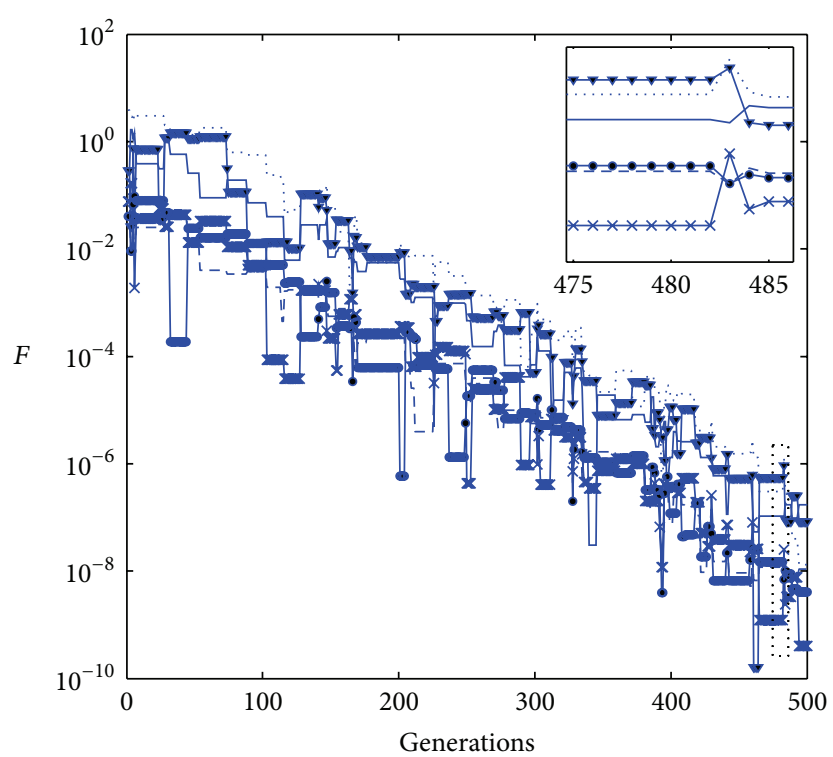

$$
\begin{array}{ll}
\cdots & |\sigma-10| \\
\longrightarrow & |\gamma-28| \\
\longrightarrow & |b-8 / 3|
\end{array}
$$

(b)

FIGURE 3: Evolution process for fractional-order Lorénz system.

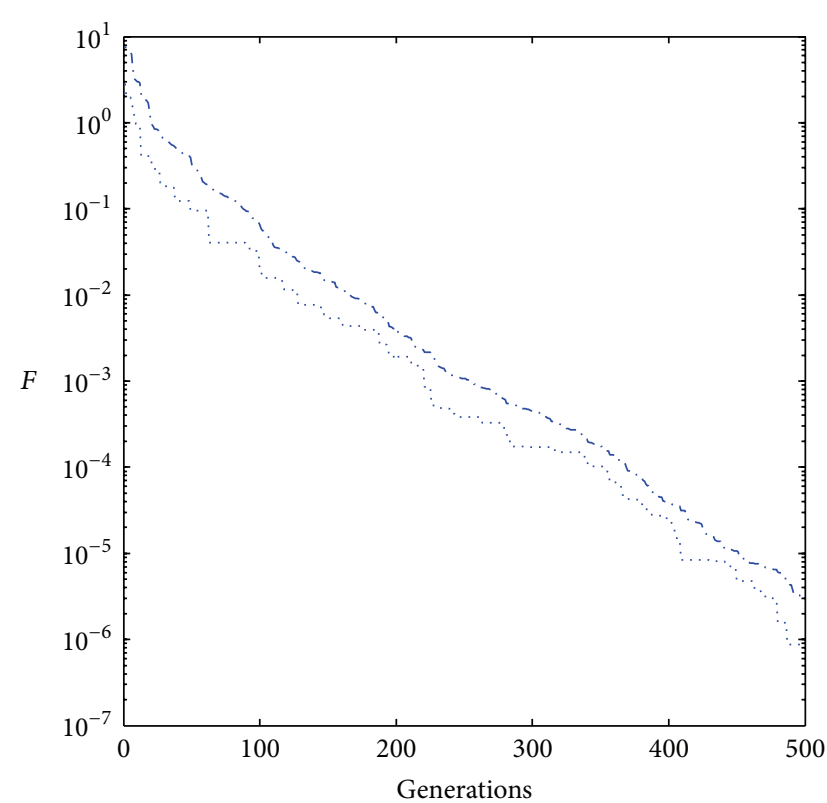

Best CSODE

-.. Mean CSODE

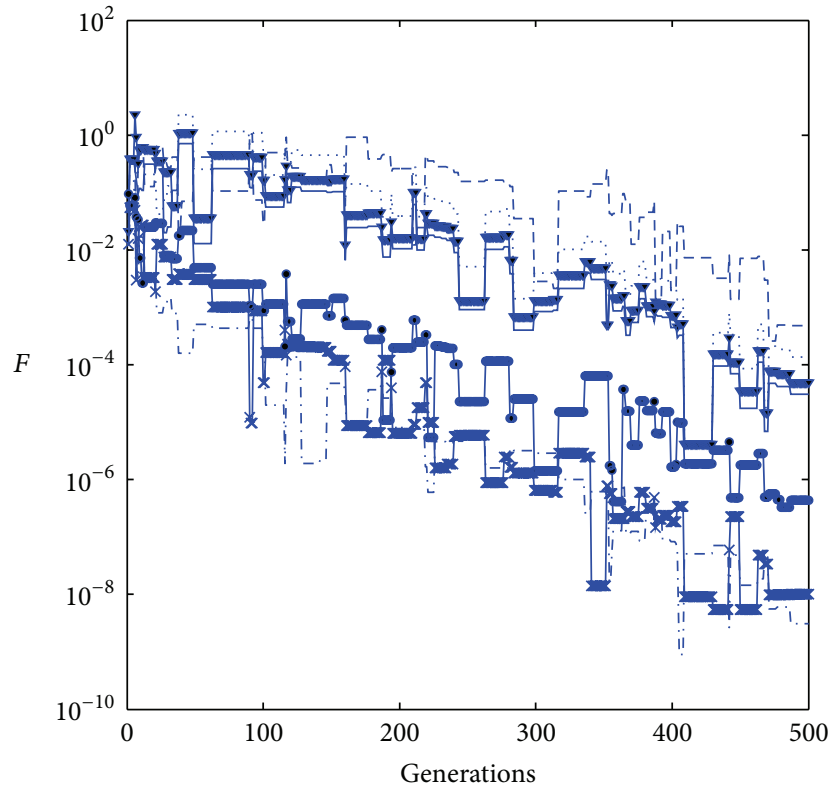

$$
\begin{array}{ll}
\ldots . .\left|\beta_{1}-(-5.5)\right| & \rightarrow\left|q_{1}-0.97\right| \\
\rightarrow-\left|\beta_{2}-3.5\right| & --\left|q_{2}-0.97\right| \\
-\left|\beta_{3}-0.8\right| & \rightarrow-\left|q_{3}-0.96\right| \\
--\left|\beta_{4}-(-1)\right| &
\end{array}
$$

(b)

FIGURE 4: Evolution process for fractional-order Arneodo system. 
TABLE 5: Simulation results for system (33).

\begin{tabular}{|c|c|c|c|c|c|c|}
\hline System (33) & $\mathrm{StD}$ & Mean & Min. & Max. & Success rate & $\mathrm{NEOF}^{\mathrm{b}}$ \\
\hline Case A & $2.3271 e+02$ & $2.2944 e+02$ & $3.4062 e-02$ & $5.2168 e+02$ & $31 \%^{\mathrm{a}}$ & 24000 \\
\hline Case $A^{c}$ & $3.5361 e+02$ & $4.3992 e+02$ & $2.9705 e-04$ & $7.2477 e+02$ & $39 \%{ }^{\mathrm{a}}$ & 24040 \\
\hline Case B & $3.3699 e-02$ & $1.6530 e-01$ & $1.5989 e-05$ & $8.3946 e-01$ & $96 \%{ }^{\mathrm{d}}$ & 23800 \\
\hline Case C & $6.2779 e-03$ & $2.4339 e-02$ & $1.4415 e-05$ & $1.7882 e-01$ & $98 \%{ }^{\mathrm{d}}$ & 39360 \\
\hline Case D & $7.5365 e-01$ & $2.3014 e+00$ & $1.1463 e-02$ & $1.6874 e+01$ & $41 \%{ }^{\mathrm{d}}$ & 48320 \\
\hline
\end{tabular}

${ }^{a}$ Success means that the solution is less than 1 in 100 independent simulations.

${ }^{\mathrm{b}}$ Number of evaluation for objective function.

${ }^{\mathrm{c}}$ By single DE with 600 generations.

${ }^{\mathrm{d}}$ Success means that the solution is less than $1 e-1$ in 100 independent simulations.

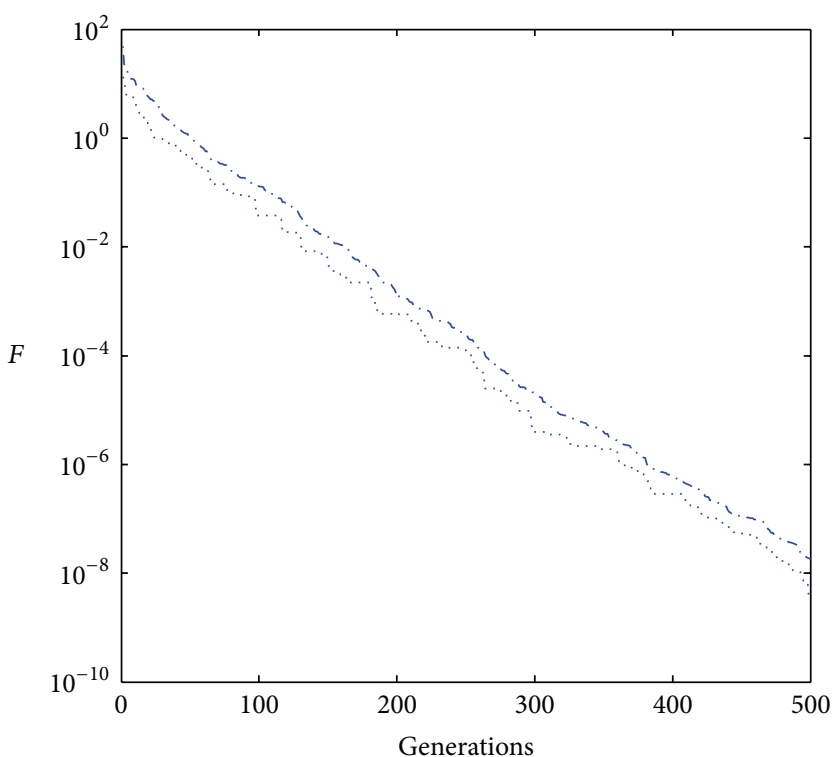

-... Best CSODE

-..- Mean CSODE

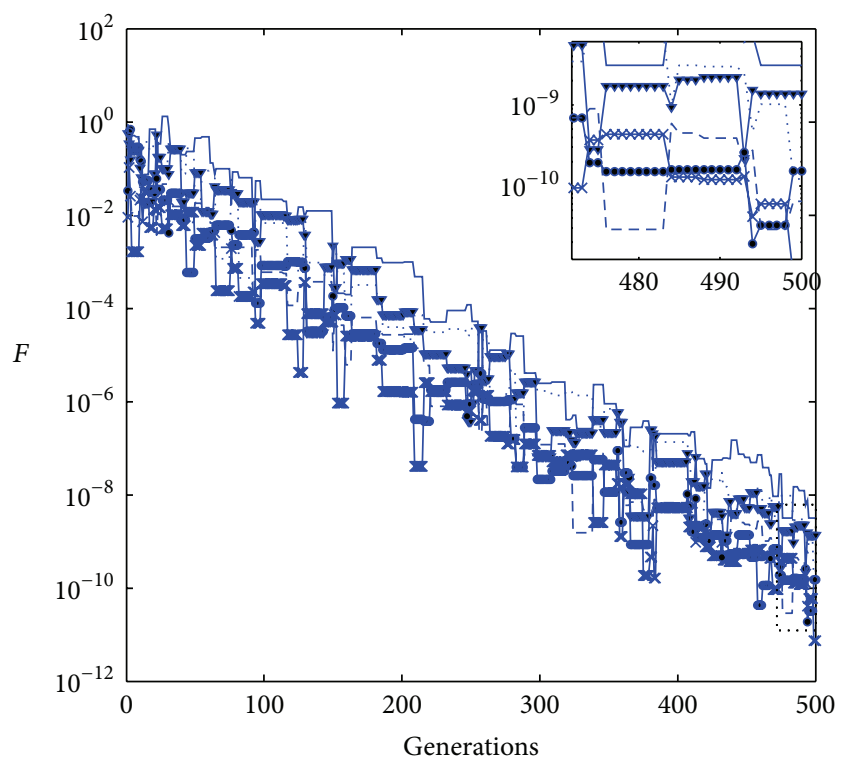

$$
\begin{aligned}
& \cdots \cdot|a-1| \\
& \rightarrow-|b-0.1| \\
& -|c-1|
\end{aligned}
$$

(a)

FIgURE 5: Evolution process for fractional-order financial system.
From the simulations results of the above fractional-order chaos system, it can be concluded that the proposed method is efficient. And from above figures, it can be concluded that the estimated systems are self-growing under the genetic operations of the proposed methods.

To test the performance of the proposed method (Algorithm 3), some more simulations are done to the fourwing incommensurate hyperfractional-order chaotic system (33) in the following cases A, B, C, and D. In these cases, each with only one condition is changed according to the original setting for system (33). The other parameters for the algorithms are the same as those for CSODE. The simulation results are listed in Table 5.

(i) Case A: enhancing the defined intervals of the unknown parameters and orders to $[0,10] \times[30,45] \times$ $[40,50] \times[0.1,1] \times[0.1,1] \times[0.1,1] \times[0.1,1]$.

(ii) Case B: reducing the number of samples for computing system (33) from 200 to 100. (iii) Case C: increasing the iteration numbers of Algorithm 3 from 500 to 800 .

(iv) Case D: changing the population size of Algorithm 3 from 40 to 80 .

Figure 12 shows the correspondent simulation results for system (33).

From results of Tables 2, 3, and 5 and Figure 12, we can conclude that minimizing the number of samples for computing the system (33) as case B, enhancing the iteration numbers as case $\mathrm{C}$, and changing the population size of Algorithm 3 as case D, will make Algorithm 3 much efficient and achieve a much more higher precision. However if the defined intervals of the unknown parameters of system (33) are enhanced, then the results will go to the opposite way. That is, the success rate is from $90 \%$ to $20 \%$ as case A.

If the number of evaluation for objective function is considered, it is that minimizing the number of samples for 


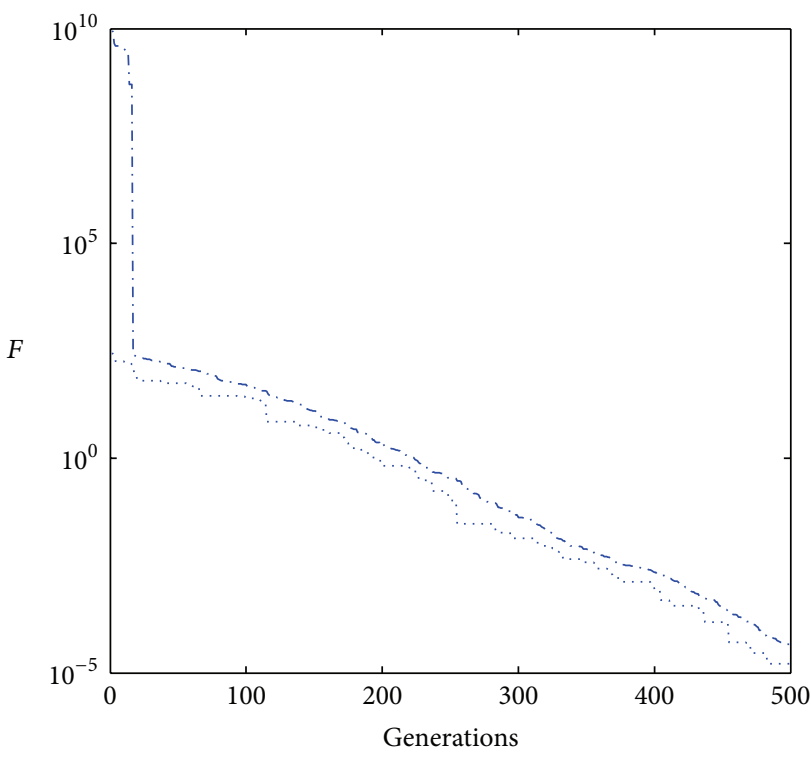

..... Best CSODE
...

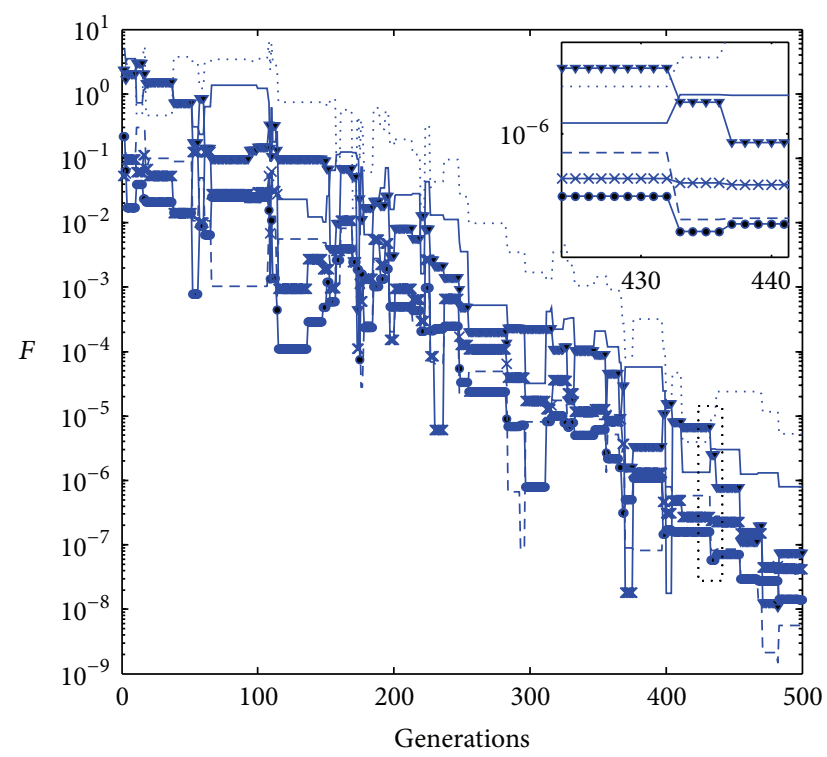

$\begin{array}{ll}\cdots|a-36| & *\left|q_{1}-0.985\right| \\ \rightarrow|b-3| & \rightarrow-\left|q_{2}-0.99\right| \\ -|c-20| & --\left|q_{3}-0.99\right|\end{array}$

(b)

FiguRE 6: Evolution process for fractional-order Lü system.
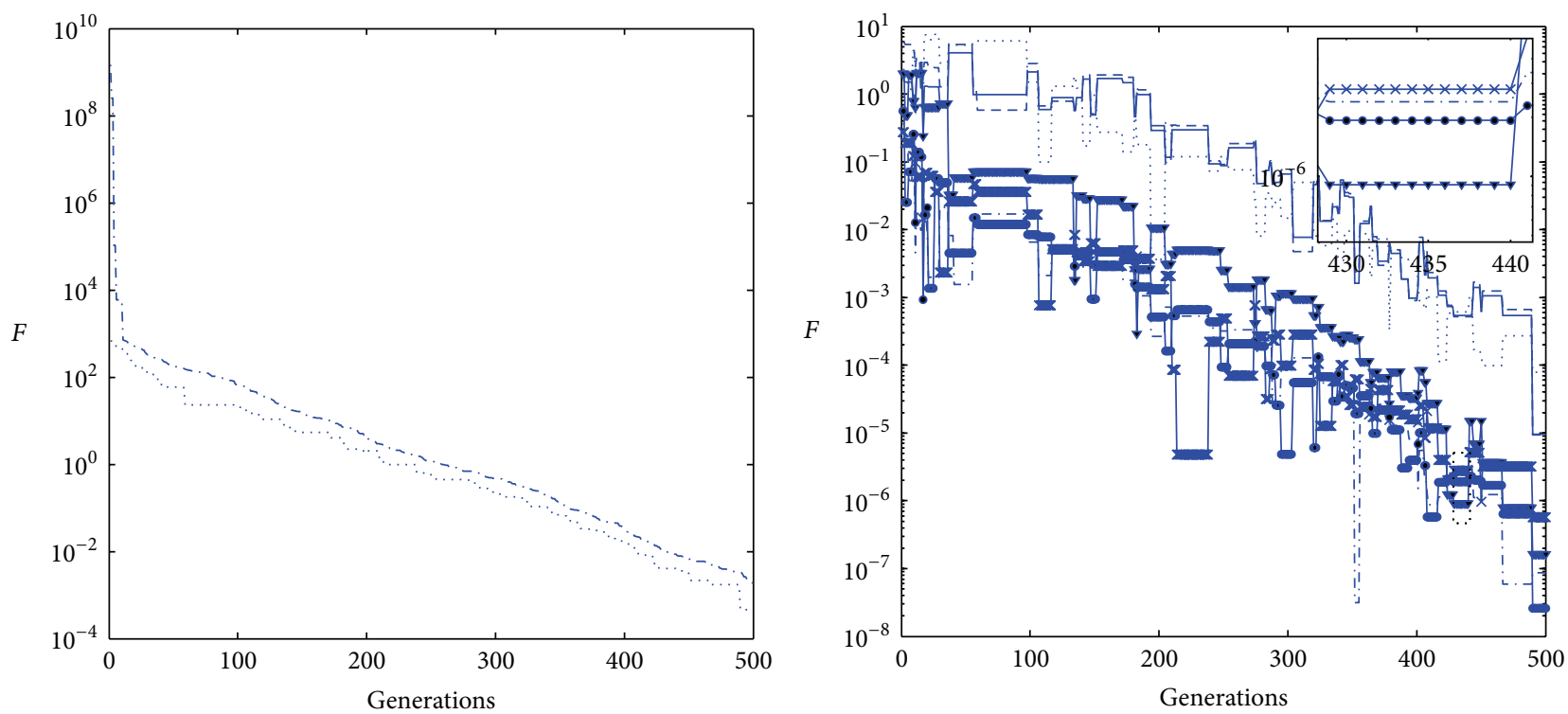

Best CSODE

-.. Mean CSODE

$$
\begin{aligned}
& \cdots|a-35| \\
& -|b-3| \\
& -|c-28| \\
& ---|d-(-7)|
\end{aligned}
$$

$\star\left|q_{1}-1\right|$

$\ldots-\left|q_{2}-1.24\right|$

$\rightarrow\left|q_{3}-1.24\right|$

(b)

FIGURE 7: Evolution process for fractional-order improper Chen system. 

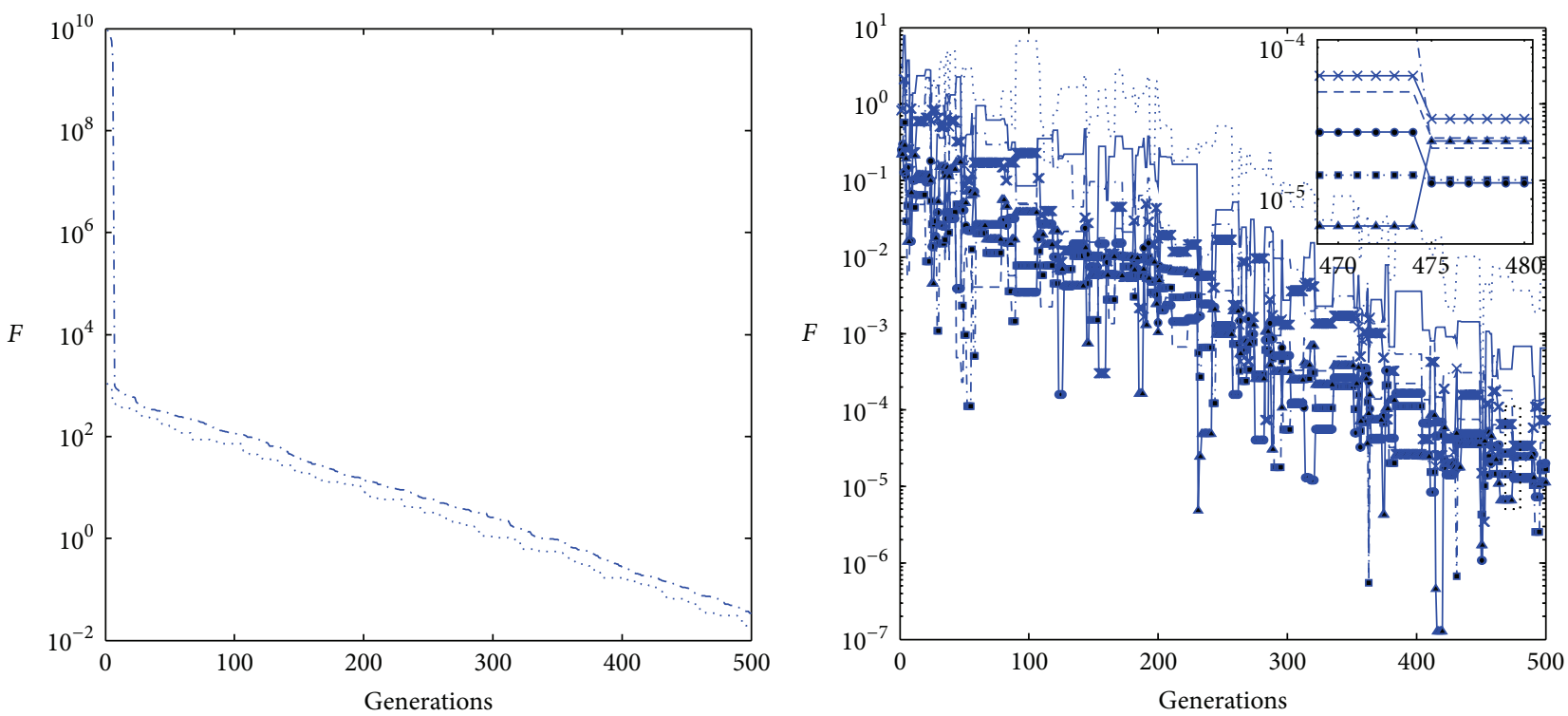

... Best CSODE

$$
\begin{aligned}
& \cdots|a-36| \\
& \cdots-|b-3| \\
& -|c-20| \\
& \times|d-1.3|
\end{aligned}
$$

$--\left|q_{1}-0.98\right|$

$\rightarrow-\left|q_{2}-0.98\right|$

$\rightarrow\left|q_{3}-0.98\right|$

$\_\left|q_{4}-0.98\right|$

(a)

(b)

Figure 8: Evolution process for hyper fractional-order Lü system.

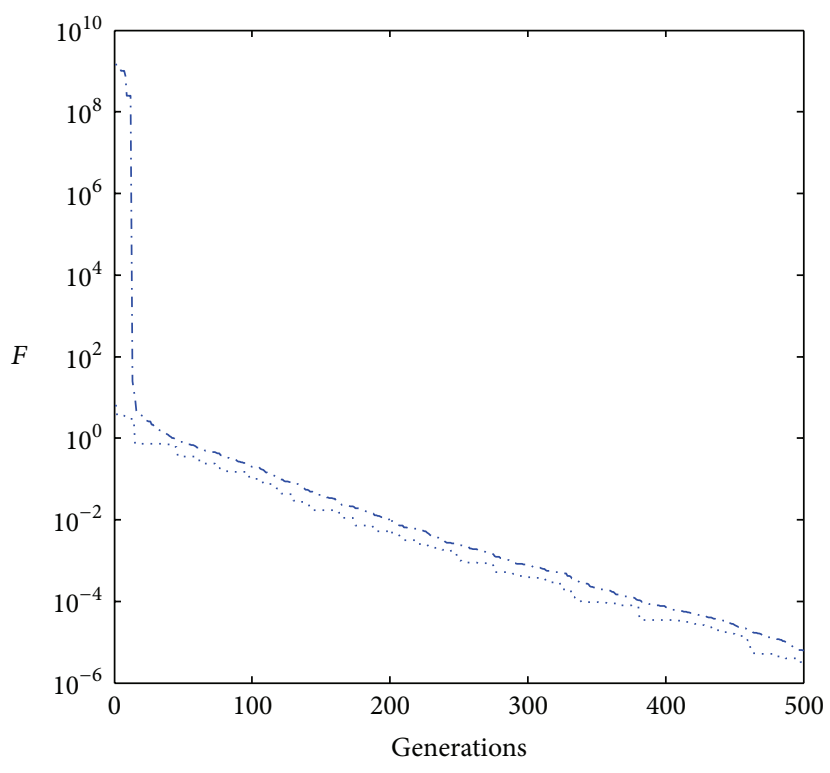

Best CSODE

...- Mean CSODE

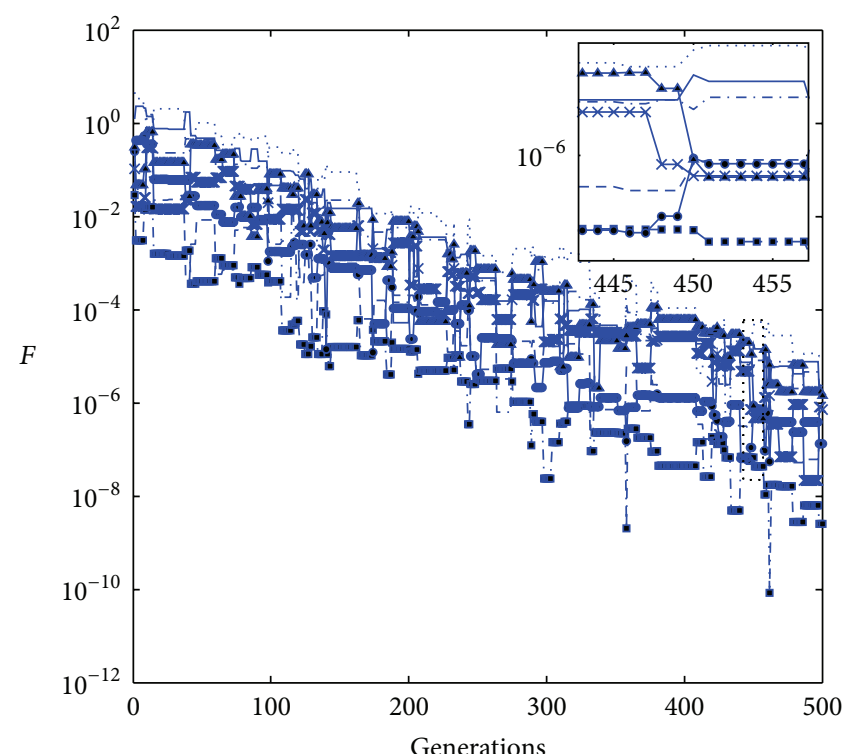

$$
\begin{aligned}
& \cdots \cdot|a-10| \\
& \cdots|b-0.1| \\
& -|c-4| \\
& -|d-0.25|
\end{aligned}
$$

(a)

$$
\begin{aligned}
& --\left|q_{1}-0.9\right| \\
& -|| q_{2}-0.9 \mid \\
& -|| q_{3}-0.9 \mid \\
& \simeq\left|q_{4}-0.9\right|
\end{aligned}
$$

(b)

FIGURE 9: Evolution process for hyper fractional-order Liu system. 


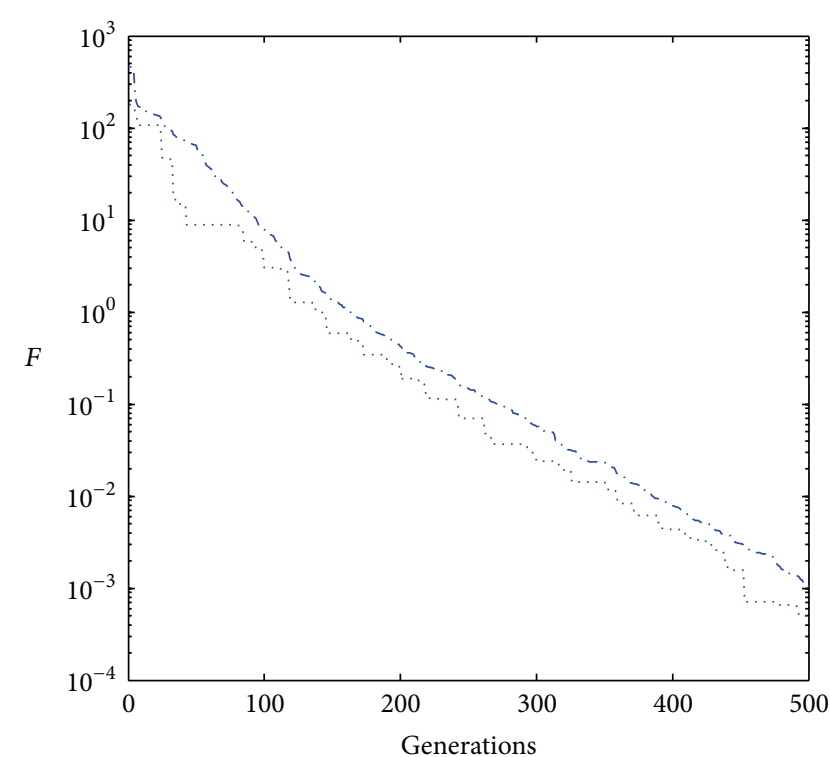

Best CSODE

... Mean CSODE

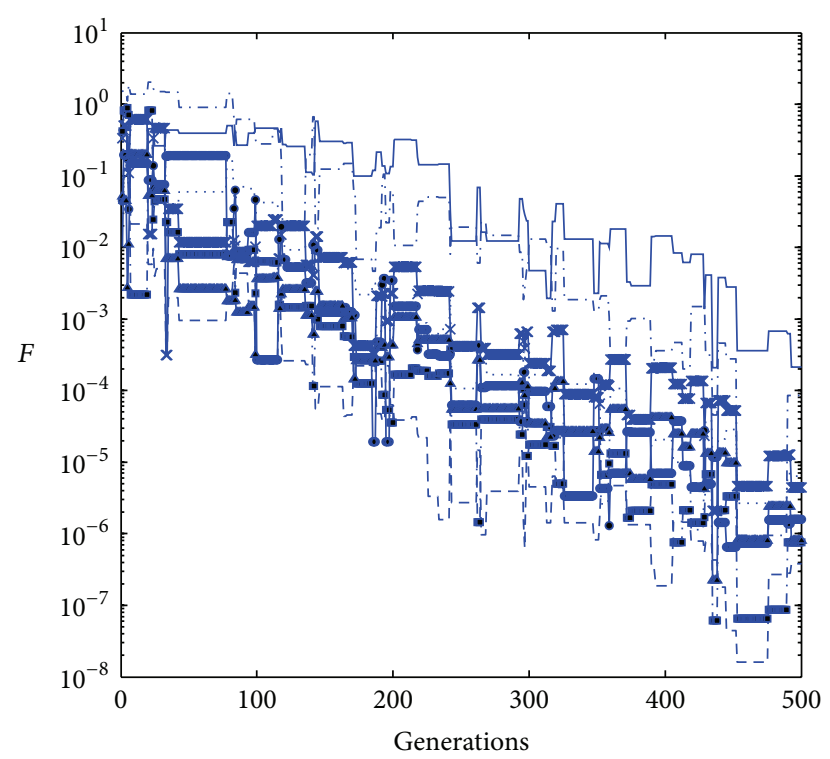

$\begin{array}{ll}\cdots \cdot & |a-32| \\ \cdots- & |b-3| \\ - & |c-0.5| \\ - & |d-0.05|\end{array}$

$---\left|q_{1}-0.95\right|$

$\rightarrow-\left|q_{2}-0.95\right|$

$\rightarrow\left|q_{3}-0.95\right|$

$\leftarrow\left|q_{4}-0.95\right|$

(a)

(b)

FIgURE 10: Evolution process for hyper fractional-order Rössler system.
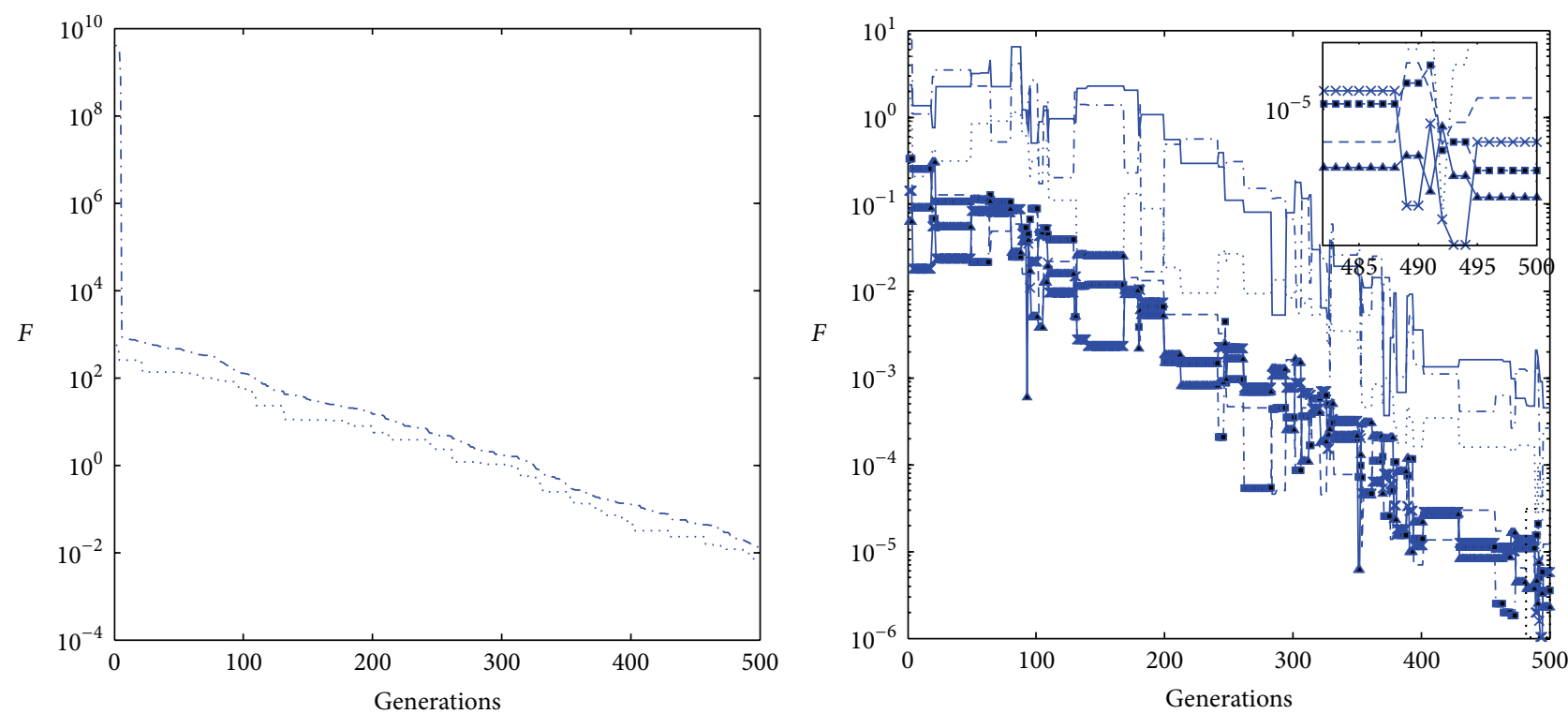

Best CSODE

$$
\begin{aligned}
& \cdots|a-8| \\
& \cdots \cdot|b-40| \\
& -\cdot|c-49| \\
& -\left|q_{1}-1\right|
\end{aligned}
$$

(a)

(b)

FIGURE 11: Evolution process for fractional-order four-wing system (33). 


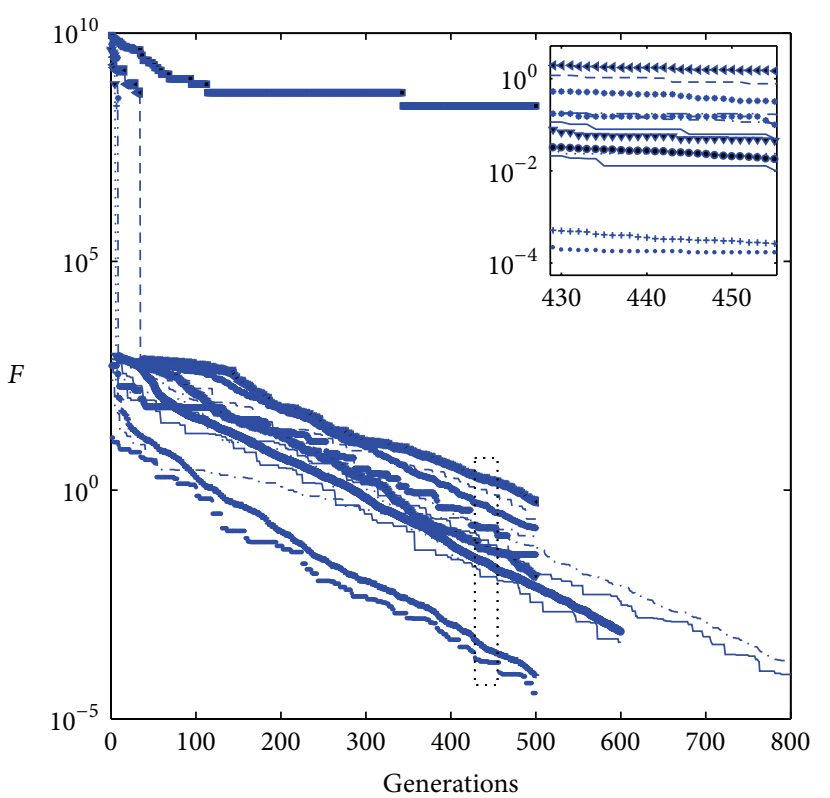

Process of one simulation

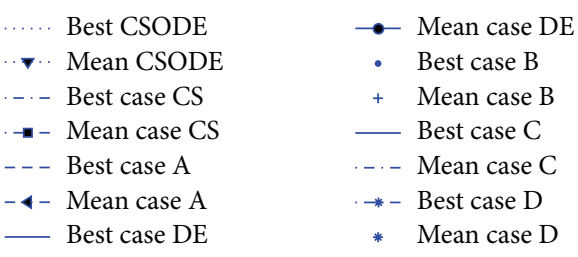

(a)

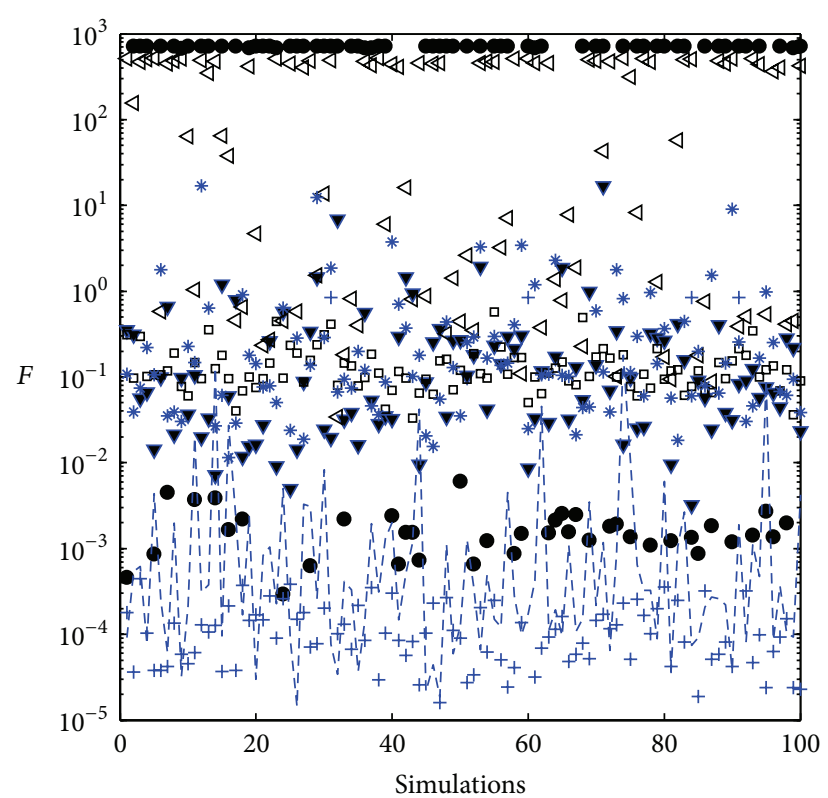

Results of $F$ for all simulations

$\begin{array}{llcl}\nabla & \text { Best } F \text { CSODE } & + & \text { Best } F \text { case B } \\ \square & \text { Best } F \text { CS } & -- & \text { Best } F \text { case C } \\ \triangleleft & \text { Best } F \text { case A } & * & \text { Best } F \text { case D } \\ - & \text { Best } F \text { case A by DE } & & \end{array}$

(b)

FIGURE 12: Simulation results for system (33).

computing the system (33) as case B is the best way to achieve higher efficiency and precision.

And according to Tables 2 and 3, Algorithm 3 based on CSODE is much better than single cuckoo search.

\section{Conclusions}

The novel Gao mathematical model in Section 2 is not only for fractional chaos parameters identification but also for reconstruction in non-Lyapunov way with three submodels $\mathrm{A}, \mathrm{B}$, and $\mathrm{C}$.

The put method based on CSODE consists of numerical optimization problem with unknown fractional-order differential equations to identify the chaotic systems. Simulation results demonstrate the effectiveness and efficiency of the proposed methods with the Gao mathematical model in Section 2. This is a novel non-Lyapunov way for fractional order chaos' unknown parameters and orders. The proposed methods solve the question that the unknown fractionalorder $q$ are not resolved in [36].

Here we have to say that this work is only about the estimation of unknown parameters and orders with the objective function (24) for noncommensurate and hyper fractional-order chaos systems in non-Lyapunov way. It can be concluded that CSODE in Algorithm 3 can be changed to other artificial intelligence methods easily. And from Tables 2, 3, 4, and 5, we can conclude that Algorithm 3 with CSODE is better than CS and GA. And using the system (33), CSODE is better than DE for the bigger scale of the unknown parameters and orders.

In the future, there are three interesting problems to be studied.

(i) Neither the fractional-orders nor some fractional order equations are unknown. That is, the objective function is chosen as (10) in the novel mathematical model in Section 2. A simple way for this might be the approaches combining the fractional orders and fractional-order equations together that might be both the estimation methods as artificial intelligent methods and the reconstruction methods as in [36] together in some degree.

(ii) Time delays and systematic parameters are unknown for fractional time-delay chaos systems. The objective function will be selected as the objective function (24) for the time-delay fractional chaotic systems as in Gao's submodel C, which have special characteristics.

(iii) Cases with noises. Normally, the white noise will be added to the Gao three sub-models. The similar ideas discussed in Section 2 will be used. 
In conclusion, it has to be stated that Algorithm 3 for fractional-order chaos systems' identification in a nonLyapunov way is a promising direction.

\section{Acknowledgments}

The work is partially supported by the NSFC Projects nos. 91324201, 81271513 of China, the Fundamental Research Funds for the Central Universities of China, the SelfDetermined and Innovative Research Funds of WUT No. 2012-Ia-035, 2012-Ia-041, 2013-Ia-040, and 2010-Ia-004, Scientific Research Foundation for Returned Scholars from Ministry of Education of China (no. 20111j0032), the HOME Program no. 11044 (Help Our Motherland through Elite Intellectual Resources from Overseas) funded by China Association for Science and Technology, the Natural Science Foundation no. 2009CBD213 of Hubei Province of China, The National Soft Science Research Program 2009GXS1D012 of China, and the National Science Foundation for Postdoctoral Scientists of China no. 20080431004. The work was partially carried out during the tenure of the ERCIM Alain Bensoussan Fellowship Programme, which is supported by the Marie Curie Cofunding of Regional, National, and International Programmes (COFUND) of the European Commission.

\section{References}

[1] R. Caponetto and S. Fazzino, "A semi-analytical method for the computation of the Lyapunov exponents of fractional-order systems," Communications in Nonlinear Science and Numerical Simulation, vol. 18, no. 1, pp. 22-27, 2013.

[2] M. S. Tavazoei and M. Haeri, "A necessary condition for double scroll attractor existence in fractional-order systems," Physics Letters A, vol. 367, no. 1-2, pp. 102-113, 2007.

[3] I. Grigorenko and E. Grigorenko, "Chaotic dynamics of the fractional lorenz system," Physical Review Letters, vol. 91, no. 42, Article ID 034101, 2003.

[4] S. K. Agrawal, M. Srivastava, and S. Das, "Synchronization of fractional order chaotic systems using active control method," Chaos, Solitons \& Fractals, vol. 45, no. 6, pp. 737-752, 2012.

[5] Q. Yang and C. Zeng, "Chaos in fractional conjugate Lorenz system and its scaling attractors," Communications in Nonlinear Science and Numerical Simulation, vol. 15, no. 12, pp. 4041-4051, 2010.

[6] K. Diethelm, N. J. Ford, and A. D. Freed, "A predictor-corrector approach for the numerical solution of fractional differential equations," Nonlinear Dynamics, vol. 29, no. 1-4, pp. 3-22, 2002.

[7] Y. Tang, X. Zhang, C. Hua, L. Li, and Y. Yang, "Parameter identification of commensurate fractional-order chaotic system via differential evolution," Physics Letters A, vol. 376, no. 4, pp. 457-464, 2012.

[8] Y. Yu, H.-X. Li, S. Wang, and J. Yu, "Dynamic analysis of a fractional-order Lorenz chaotic system," Chaos, Solitons \& Fractals, vol. 42, no. 2, pp. 1181-1189, 2009.

[9] L.-G. Yuan and Q.-G. Yang, "Parameter identification and synchronization of fractional-order chaotic systems," Communications in Nonlinear Science and Numerical Simulation, vol. 17, no. 1, pp. 305-316, 2012.
[10] J. B. Hu, J. Xiao, and L. D. Zhao, "Synchronizing an improper fractional Chen chaotic system," Journal of Shanghai University. Natural Science, vol. 17, no. 6, pp. 734-739, 2011.

[11] C. Li and G. Peng, "Chaos in Chen's system with a fractional order," Chaos, Solitons \& Fractals, vol. 22, no. 2, pp. 443-450, 2004.

[12] C. Li and G. Chen, "Chaos and hyperchaos in the fractionalorder Rössler equations," Physica A, vol. 341, no. 1-4, pp. 55-61, 2004.

[13] W. Deng, E. Shulin, D. Sun, P. Wang, D. Zhang, and W. Xu, "Fabrication of vertical coupled polymer microring resonator," in ICO20: Optical Communication, Y.-C. Chung and S. Xie, Eds., vol. 6025 of Proceedings of SPIE, pp. 334-339, 2006.

[14] L. Song, J. Yang, and S. Xu, "Chaos synchronization for a class of nonlinear oscillators with fractional order," Nonlinear Analysis: Theory, Methods and Applications, vol. 72, no. 5, pp. 2326-2336, 2010.

[15] R.-X. Zhang, Y. Yang, and S.-P. Yang, "Adaptive synchronization of the fractional-order unified chaotic system," Acta Physica Sinica, vol. 58, no. 9, pp. 6039-6044, 2009.

[16] W. H. Deng and C. P. Li, "Chaos synchronization of the fractional Lü system," Physica A, vol. 353, no. 1-4, pp. 61-72, 2005.

[17] H. Deng, T. Li, Q. Wang, and H. Li, "A fractional-order hyperchaotic system and its synchronization," Chaos, Solitons \& Fractals, vol. 41, no. 2, pp. 962-969, 2009.

[18] C. P. Li, W. H. Deng, and D. Xu, "Chaos synchronization of the Chua system with a fractional order," Physica A, vol. 360, no. 2, pp. 171-185, 2006.

[19] W. Deng and J. Lü, "Generating multi-directional multi-scroll chaotic attractors via a fractional differential hysteresis system," Physics Letters A, vol. 369, no. 5-6, pp. 438-443, 2007.

[20] Z. M. Odibat, N. Corson, M. A. Aziz-Alaoui, and C. Bertelle, "Synchronization of chaotic fractional-order systems via linear control," International Journal of Bifurcation and Chaos, vol. 20, no. 1, pp. 81-97, 2010.

[21] Z. Odibat, "A note on phase synchronization in coupled chaotic fractional order systems," Nonlinear Analysis: Real World Applications, vol. 13, no. 2, pp. 779-789, 2012.

[22] X. Wu, D. Lai, and H. Lu, "Generalized synchronization of the fractional-order chaos in weighted complex dynamical networks with nonidentical nodes," Nonlinear Dynamics, vol. 69, no. 1-2, pp. 667-683, 2012.

[23] K. Sun and J. C. Sprott, "Bifurcations of fractional-order diffusionless lorenz system," Electronic Journal of Theoretical Physics, vol. 6, no. 22, pp. 123-134, 2009.

[24] X.-J. Wu and S.-L. Shen, "Chaos in the fractional-order Lorenz system," International Journal of Computer Mathematics, vol. 86, no. 7, pp. 1274-1282, 2009.

[25] E. Kaslik and S. Sivasundaram, "Analytical and numerical methods for the stability analysis of linear fractional delay differential equations," Journal of Computational and Applied Mathematics, vol. 236, no. 16, pp. 4027-4041, 2012.

[26] S. Bhalekar and V. Daftardar-Gejji, "Fractional ordered Liu system with time-delay," Communications in Nonlinear Science and Numerical Simulation, vol. 15, no. 8, pp. 2178-2191, 2010.

[27] J. G. Lu and G. Chen, "A note on the fractional-order Chen system," Chaos, Solitons \& Fractals, vol. 27, no. 3, pp. 685-688, 2006.

[28] Y. Al-Assaf, R. El-Khazali, and W. Ahmad, "Identification of fractional chaotic system parameters," Chaos, Solitons \& Fractals, vol. 22, no. 4, pp. 897-905, 2004. 
[29] E. H. Doha, A. H. Bhrawy, and S. S. Ezz-Eldien, "A new Jacobi operational matrix: an application for solving fractional differential equations," Applied Mathematical Modelling, vol. 36, no. 10, pp. 4931-4943, 2012.

[30] G. Si, Z. Sun, Y. Zhang, and W. Chen, "Projective synchronization of different fractional-order chaotic systems with nonidentical orders," Nonlinear Analysis: Real World Applications, vol. 13, no. 4, pp. 1761-1771, 2012.

[31] K. Diethelm and N. J. Ford, "Analysis of fractional differential equations," Journal of Mathematical Analysis and Applications, vol. 265, no. 2, pp. 229-248, 2002.

[32] W. Deng, C. Li, and J. Lü, "Stability analysis of linear fractional differential system with multiple time delays," Nonlinear Dynamics, vol. 48, no. 4, pp. 409-416, 2007.

[33] M. S. Tavazoei and M. Haeri, "Chaotic attractors in incommensurate fractional order systems," Physica D, vol. 237, no. 20, pp. 2628-2637, 2008.

[34] G. Si, Z. Sun, H. Zhang, and Y. Zhang, "Parameter estimation and topology identification of uncertain fractional order complex networks," CommuNications in Nonlinear Science and Numerical Simulation, vol. 17, no. 12, pp. 5158-5171, 2012.

[35] G. Si, Z. Sun, H. Zhang, and Y. Zhang, "Parameter estimation and topology identification of uncertain fractional order complex networks," Communications in Nonlinear Science and Numerical Simulation, vol. 17, no. 12, pp. 5158-5171, 2012.

[36] F. Gao, F.-X. Fei, X.-J. Lee, H.-Q. Tong, Y.-F. Deng, and H.-L. Zhao, "Inversion mechanism with functional extrema model for identification incommensurate and hyper fractional chaos via differential evolution," Expert Systems with Applications, vol. 41, no. 4(2), pp. 1915-1927, 2014.

[37] I. Publications, Ed., Cuckoo Search via Levy Ights, 2009.

[38] X.-S. Yang and S. Deb, "Engineering optimisation by cuckoo search," International Journal of Mathematical Modelling and Numerical Optimisation, vol. 1, no. 4, pp. 330-343, 2010.

[39] X.-S. Yang and S. Deb, "Multiobjective cuckoo search for design optimization," Computers \& Operations Research, vol. 40, no. 6, pp. 1616-1624, 2013.

[40] C. Li and C. Tao, "On the fractional Adams method," Computers and Mathematics with Applications, vol. 58, no. 8, pp. 1573-1588, 2009.

[41] K. Diethelm, "An efficient parallel algorithm for the numerical solution of fractional differential equations," Fractional Calculus and Applied Analysis, vol. 14, no. 3, pp. 475-490, 2011.

[42] A. A. Kilbas, H. M. Srivastava, and J. J. Trujillo, Theory and Applications of Fractional Differential Equations, vol. 204 of North-Holland Mathematics Studies, North-Holland, Amsterdam, The Netherlands, 2006.

[43] K. S. Miller and B. Ross, An Introduction to the Fractional Calculus and Fractional Differential Equations, John Wiley \& Sons, New York, NY, USA, 1993.

[44] S. G. Samko, A. A. Kilbas, and O. I. Marichev, Fractional Integrals and Derivatives: Theory and Applications, Gordon and Breach Science Publishers, Yverdon, Switzerland, 1993.

[45] K. B. Oldham and J. Spanier, The Fractional Calculus, vol. 111 of Mathematics in Science and Engineering, Academic Press, New York, NY, USA, 1974.

[46] I. Podlubny, Fractional Differential Equations: An Introduction to Fractional Derivatives, Fractional Differential Equations, vol. 198 of Mathematics in Science and Engineering, Academic Press, San Diego, Calif, USA, 1999.
[47] I. Petráš, "Fractional-order chaotic systems," in FractionalOrder Nonlinear Systems, Nonlinear Physical Science, pp. 103184, Springer, Berlin, Germany, 2011.

[48] I. Petráš, "Fractional calculus," in Nonlinear Physica, pp. 7-42, Springer, Berlin, Germany, 2011.

[49] J. Koza, Genetic Programming: On the Programming of Computers by Means of Natural Selection, Complex Adaptive Systems, MIT Press, Cambridge, MA, USA, 1992.

[50] J. Koza, Genetic Programming II: Automatic Discovery of Reusable Programs, MIT Press, 1994.

[51] R. Poli, W. Langdon, and N. McPhee, A Field Guide to Genetic Programming, Lulu Enterprises, Essex, UK, 2008.

[52] A. H. Gandomi and A. H. Alavi, "A new multi-gene genetic programming approach to nonlinear system modeling. Part I: materials and structural engineering problems," Neural Computing \& Applications, vol. 21, no. 1, pp. 171-187, 2012.

[53] A. H. Gandomi and A. H. Alavi, "A new multi-gene genetic programming approach to non-linear system modeling. Part II: geotechnical and earthquake engineering problems," Neural Computing \& Applications, vol. 21, no. 1, pp. 189-201, 2012.

[54] Y. Ikeda, "Estimation of chaotic ordinary differential equations by coevolutional genetic programming," Electronics and Communications in Japan, Part III, vol. 86, no. 2, pp. 1-12, 2003.

[55] S. J. Kirstukas, K. M. Bryden, and D. A. Ashlock, "A hybrid genetic programming approach for the analytical solution of differential equations," International Journal of General Systems, vol. 34, no. 3, pp. 279-299, 2005.

[56] V. Varadan and H. Leung, "Chaotic system reconstruction from noisy time series measurements using improved least squares genetic programming," in IEEE International Symposium on Circuits and Systems (ISCAS '02), vol. 3, pp. 65-68, May 2002.

[57] W. Zhang, Z.-M. Wu, and G.-K. Yang, "Genetic programmingbased chaotic time series modeling," Journal of Zhejiang University: Science, vol. 5, no. 11, pp. 1432-1439, 2004.

[58] W. Zhang, G.-K. Yang, and Z.-M. Wu, "Genetic programmingbased modeling on chaotic time series," in Proceedings of International Conference on Machine Learning and Cybernetics, vol. 4, pp. 2347-2352, August 2004.

[59] V. Varadan and H. Leung, "Reconstruction of polynomial systems from noisy time-series measurements using genetic programming," IEEE Transactions on Industrial Electronics, vol. 48, no. 4, pp. 742-748, 2001.

[60] I. Zelinka and A. Raidl, "Evolutionary reconstruction of chaotic systems," in Studies in Computational Intelligence, vol. 267, chapter 8, pp. 265-291, Springer, 2010.

[61] I. Zelinka, D. Davendra, R. Senkerik, and R. Jasek, "An investigation on evolutionary identification of continuous chaotic systems," in Proceedings of the 4th Global Conference on Power Control and Optimization, AIP Conference Series, pp. 280-284, December 2010.

[62] I. Zelinka, M. Chadli, D. Davendra, R. Senkerik, and R. Jasek, "An investigation on evolutionary reconstruction of continuous chaotic systems," Mathematical and Computer Modelling, vol. 57, no. 1-2, pp. 2-15, 2011.

[63] F. Gao, J.-J. Lee, Z. Li, H. Tong, and X. Lü, "Parameter estimation for chaotic system with initial random noises by particle swarm optimization," Chaos, Solitons \& Fractals, vol. 42, no. 2, pp. 1286-1291, 2009.

[64] F. Gao, Y. Qi, I. Balasingham, Q. Yin, and H. Gao, "A Novel non-Lyapunov way for detecting uncertain parameters of chaos system with random noises," Expert Systems with Applications, vol. 39, no. 2, pp. 1779-1783, 2012. 
[65] X.-P. Guan, H.-P. Peng, L.-X. Li, and Y.-Q. Wang, "Parameters identification and control of Lorenz chaotic system," Acta Physica Sinica, vol. 50, no. 1, p. 29, 2001.

[66] U. Parlitz, "Estimating model parameters from time series by autosynchronization," Physical Review Letters, vol. 76, no. 8, pp. 1232-1235, 1996.

[67] L. Li, Y. Yang, H. Peng, and X. Wang, "Parameters identification of chaotic systems via chaotic ant swarm," Chaos, Solitons \& Fractals, vol. 28, no. 5, pp. 1204-1211, 2006.

[68] W.-D. Chang, "Parameter identification of Chen and Lü systems: a differential evolution approach," Chaos, Solitons \& Fractals, vol. 32, no. 4, pp. 1469-1476, 2007.

[69] J.-F. Chang, Y.-S. Yang, T.-L. Liao, and J.-J. Yan, "Parameter identification of chaotic systems using evolutionary programming approach," Expert Systems with Applications, vol. 35, no. 4, pp. 2074-2079, 2008.

[70] F. Gao, Z.-Q. Li, and H.-Q. Tong, "Parameters estimation online for Lorenz system by a novel quantum-behaved particle swarm optimization," Chinese Physics B, vol. 17, no. 4, pp. 1196-1201, 2008.

[71] F. Gao and H.-Q. Tong, "Parameter estimation for chaotic system based on particle swarm optimization," Acta Physica Sinica, vol. 55, no. 2, pp. 577-582, 2006.

[72] L. Shen and M. Wang, "Robust synchronization and parameter identification on a class of uncertain chaotic systems," Chaos, Solitons \& Fractals, vol. 38, no. 1, pp. 106-111, 2008.

[73] K. Yang, K. Maginu, and H. Nomura, "Parameters identification of chaotic systems by quantum-behaved particle swarm optimization," International Journal of Computer Mathematics, vol. 86, no. 12, pp. 2225-2235, 2009.

[74] F. Gao, Y. Qi, Q. Yin, and J. Xiao, "Solving problems in chaos control though an differential evolution algorithm with region zooming," Applied Mechanics and Materials, vol. 110-116, pp. 5048-5056, 2012.

[75] F. Gao, F.-X. Fei, Q. Xu, Y.-F. Deng, Y.-B. Qi, and I. Balasingham, "A novel artificial bee colony algorithm with space contraction for unknown parameters identification and time-delays of chaotic systems," Applied Mathematics and Computation, vol. 219, no. 2, pp. 552-568, 2012.

[76] E. N. Lorénz, "Deterministic nonperiodic flow," Journal of the Atmospheric Sciences, vol. 20, no. 2, pp. 130-141, 1963.

[77] J. G. Lu, "Chaotic dynamics of the fractional-order Lü system and its synchronization," Physics Letters A, vol. 354, no. 4, pp. 305-311, 2006.

[78] C. Li and G. Chen, "Chaos in the fractional order Chen system and its control," Chaos, Solitons \& Fractals, vol. 22, no. 3, pp. 549-554, 2004.

[79] R. Storn and K. Price, "Differential evolution-a simple and efficient heuristic for global optimization over continuous spaces," Journal of Global Optimization, vol. 11, no. 4, pp. 341359, 1997.

[80] F. Gao and H. Tong, "Computing two linchpins of topological degree by a novel differential evolution algorithm," International Journal of Computational Intelligence and Applications, vol. 5, no. 3, pp. 335-350, 2005.

[81] F. Gao, "Computing unstable Period orbits of discrete chaotic system though differential evolutionary algorithms basing on elite subspace," System Engineering Theory and Practice, vol. 25, no. 4, pp. 96-102, 2005.

[82] C.-W. Chiang, W.-P. Lee, and J.-S. Heh, "A 2-Opt based differential evolution for global optimization," Applied Soft Computing Journal, vol. 10, no. 4, pp. 1200-1207, 2010.
[83] K. V. Price, R. M. Storn, and J. A. Lampinen, Differential Evolution: A Practical Approach to Global Optimization, Natural Computing Series, Springer, Berlin, Germany, 2005.

[84] J. G. Lu, "Chaotic dynamics and synchronization of fractionalorder Arneodo's systems," Chaos, Solitons \& Fractals, vol. 26, no. 4, pp. 1125-1133, 2005.

[85] J.-G. Lu, "Chaotic dynamics and synchronization of fractionalorder Genesio-Tesi systems," Chinese Physics, vol. 14, no. 8, pp. 1517-1521, 2005.

[86] I. Petráš, "A note on the fractional-order Chua's system," Chaos, Solitons \& Fractals, vol. 38, no. 1, pp. 140-147, 2008.

[87] L.-D. Zhao, J.-B. Hu, and X.-H. Liu, "Adaptive tracking control and synchronization of fractional hyper-chaotic Lorenz system with unknown parameters," Acta Physica Sinica, vol. 59, no. 4, pp. 2305-2309, 2010.

[88] F.-H. Min, Y. Yu, and C.-J. Ge, "Circuit implementation and tracking control of the fractional-order hyper-chaotic Lü system," Acta Physica Sinica, vol. 58, no. 3, pp. 1456-1461, 2009.

[89] C.-X. Liu, "A hyperchaotic system and its fractional order circuit simulation," Acta Physica Sinica, vol. 56, no. 12, pp. 6865-6873, 2007.

[90] D. Li, L.-M. Deng, Y.-X. Du, and Y.-Y. Yang, "Synchronization for fractional order hyperchaotic chen system and fractional order hyperchaotic rössler system with different structure," Acta Physica Sinica, vol. 61, no. 5, Article ID 050502, 2012.

[91] R.-X. Zhang and S.-P. Yang, "Modified adaptive controller for synchronization of incommensurate fractional-order chaotic systems," Chinese Physics B, vol. 21, no. 3, Article ID 030505, 2012.

[92] S. Dadras, H. R. Momeni, G. Qi, and Z.-L. Wang, "Fourwing hyperchaotic attractor generated from a new $4 \mathrm{D}$ system with one equilibrium and its fractional-order form," Nonlinear Dynamics, vol. 67, no. 2, pp. 1161-1173, 2012. 


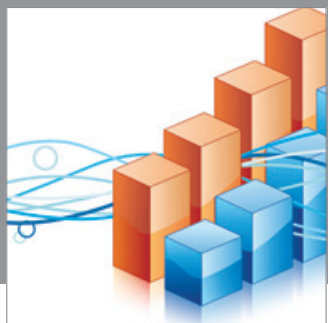

Advances in

Operations Research

mansans

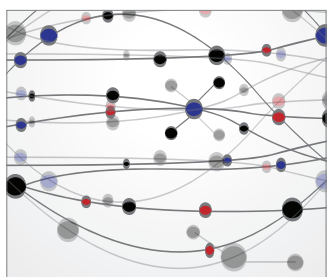

The Scientific World Journal
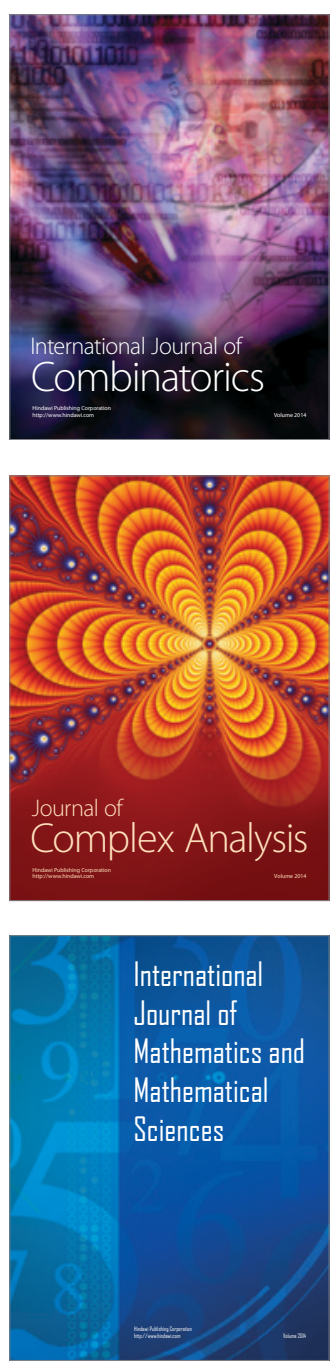
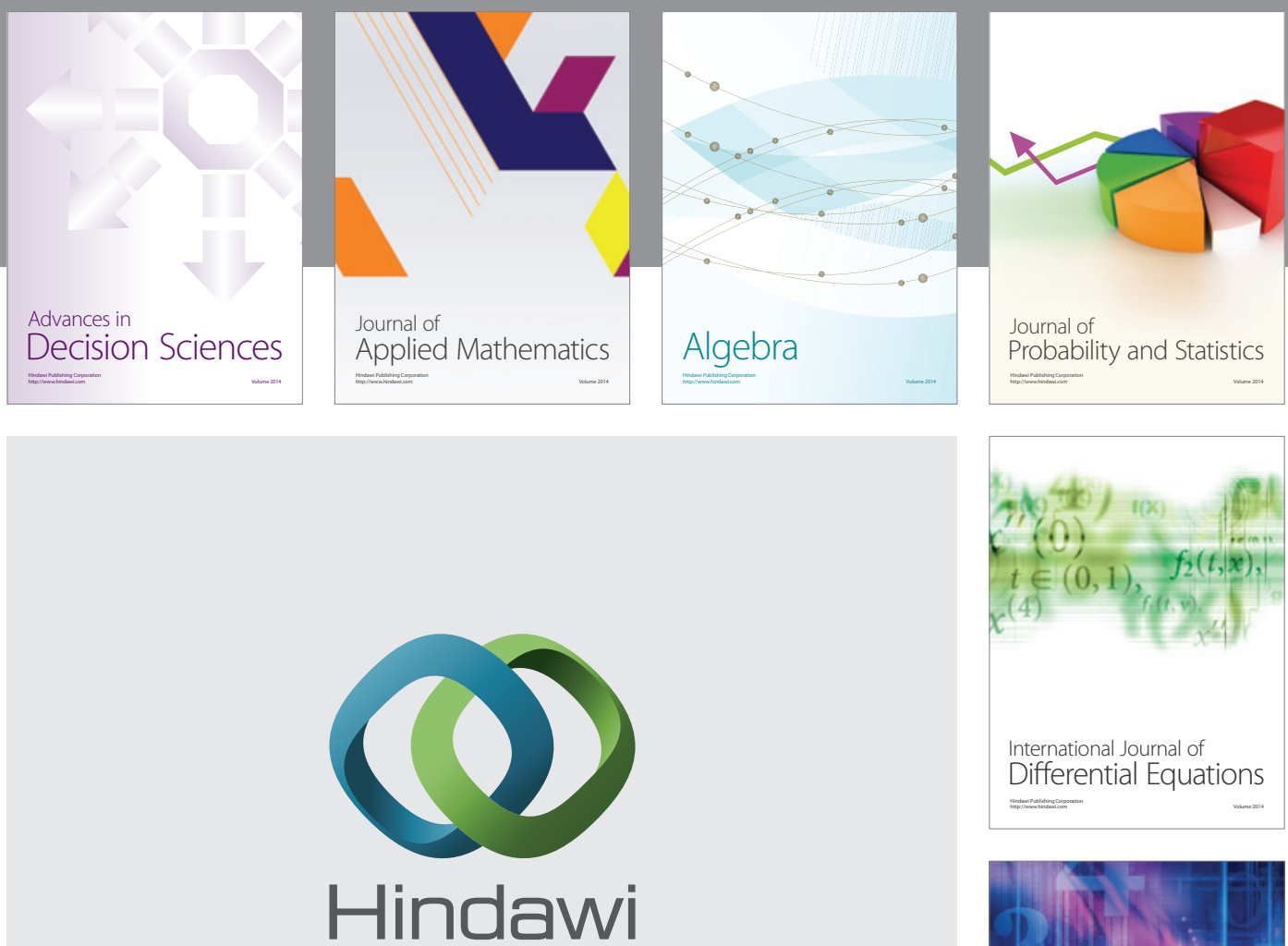

Submit your manuscripts at http://www.hindawi.com
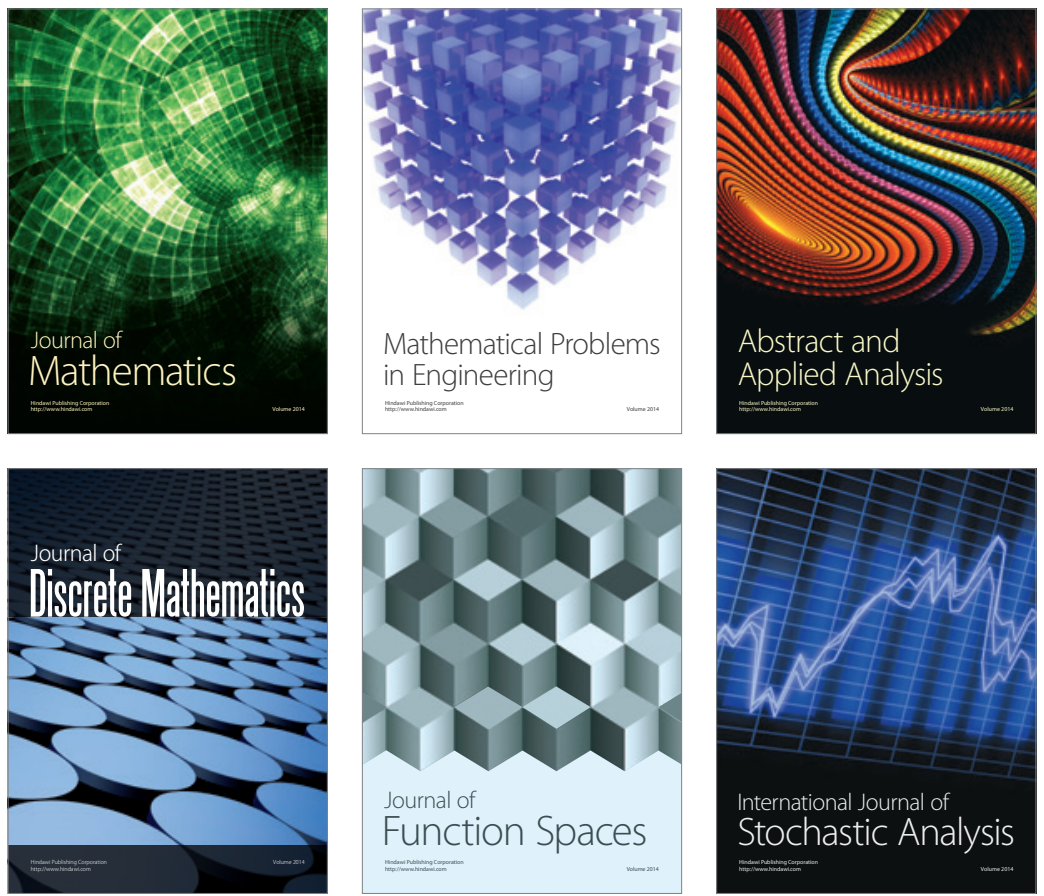

Journal of

Function Spaces

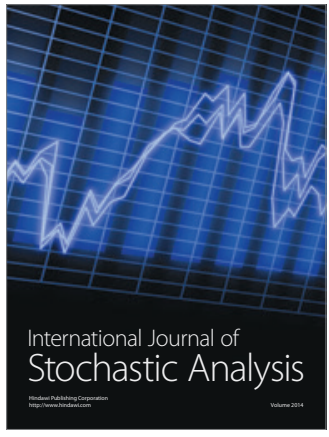

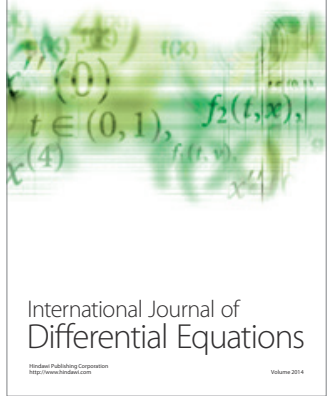
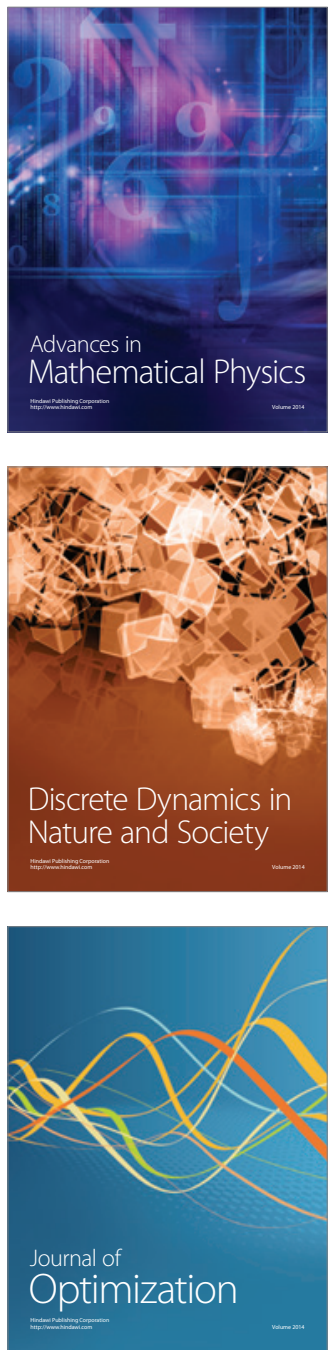